\title{
Hysteresis effects of three-phase relative permeabilities on black-oil reservoir simulation under WAG injection protocols
}

\author{
Ehsan Ranaee ${ }^{\mathrm{a}, *}$, Fabio Inzoli ${ }^{\mathrm{a}}$, Monica Riva ${ }^{\mathrm{b}, \mathrm{c}}$, Alberto Guadagnini ${ }^{\mathrm{b}, \mathrm{c}}$ \\ a Dipartimento di Energia, Politecnico di Milano, Via Lambruschini 4, 20156, Milano, Italy \\ b Dipartimento di Ingegneria Civile e Ambientale, Politecnico di Milano, Piazza L. Da Vinci 32, 20133, Milano, Italy

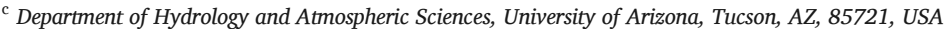

\section{ART ICLE INFO}

Keywords:

Relative permeability

Hysteresis effects

Black-oil reservoir simulation

WAG injection

Well control

\begin{abstract}
A B S T R A C T
We study the impact of explicitly including hysteresis effects (HEs) for relative permeabilities in numerical simulations of enhanced oil recovery approaches grounded on the application of Water Alternate Gas (WAG) injection protocols. Our work explores the significance of hysteresis exhibited by the relative permeability of the non-wetting and/or intermediate wetting phases on the results of reservoir simulations performed within a black-oil modeling approach. We consider four diverse strategies that can be employed to model relative permeabilities of fluid phases under WAG scenarios implemented in a water-wet system following primary waterflooding. The effects of all these modeling strategies are assessed in terms of field oil efficiency (FOE) and gas oil ratio (FGOR) evaluated at the total production lifetime of the reservoir, as compared against a base case where only waterflooding is applied to promote oil recovery. Two of these strategies are implemented and used in typical commercial black-oil reservoir simulators and either neglect hysteresis or consider it only for the gas phase. We then examine a procedure where only hysteresis for relative permeability of the oil phase is considered. Finally, we explicitly consider hysteresis of both non- and intermediate-wetting phases. Our results generally show that including HEs yields an increased FOE and a decreased FGOR. This is consistent with the observation that when injection well controls are set at an optimum value, a non-negligible portion of the domain is associated with saturation paths corresponding to three-phase flow conditions, where saturation history can play a significant role. As compared against a base case where waterflooding is considered as the sole strategy for oil recovery, including hysteresis on three-phase relative permeabilities in the numerical simulations of WAG promotes an increased net present value. We also analyze the impact on reservoir simulation responses of the main WAG injection parameters, i.e., (i) injection rate of gas and water, and duration of (ii) WAG cycles, and (iii) primary waterflooding.
\end{abstract}

\section{Introduction}

Water-Alternating-Gas (WAG) injection is considered as a viable tertiary oil recovery technique, with already significant successful applications (Christensen et al., 2001; Afzali et al., 2018 and references therein). WAG techniques date to 1957, with an application to a sandstone reservoir in Alberta (Canada) aimed at controlling the mobility of the gas phase during gas injection (Afzali et al., 2018). Due to the low gas viscosity and considerable density difference between the gas and reservoir crude oil, gas injection processes exhibit a poor sweep efficiency at the microscopic level, thus resulting in effects such as (a) bypassing a portion of the oil, (b) fluid front instability, and/or (c) early breakthrough in the swept area of a reservoir (Juanes and Blunt, 2006). Protocols associated with water alternating gas slugs can be a solution to these issues.

Similar to other flooding techniques, the performance of WAG can be measured not only in terms of the macroscopic (or volumetric) efficiency, but also considering the microscopic (i.e., displacement at the pore scale level) efficiency of the flooding process (Speight, 2009). A WAG injection scheme aims at combining high macroscopic sweep efficiency of the waterflooding operation with high displacement efficiency of the gas injection process to improve oil recovery (Kulkarni and Rao, 2005). Field implementation of WAG are reported to achieve (on average) around 10\% improvement of oil recovery, when compared to conventional oil recovery techniques. In this context, CO2-

\footnotetext{
* Corresponding author.

Email address: ehsan.ranaee@polimi.it (E. Ranaee)
} 
WAG is also subject to increased attention due to concerns about global change and environmental issues (Afzali et al., 2018 and references therein).

This study is focused on black-oil simulators, which are widely used in the context of reservoir simulation applications because of their potential to obtain acceptable results with relatively low computational costs in cases where compositional effects are negligible (Ertekin et al., 2001; Khorsandi et al., 2018). Simulation of three-phase fluid flow in black-oil models takes into account the occurrence of mass exchange between oil and gas phases, according to the following set of equations (e.g., Chen et al., 2006)

$\frac{\partial}{\partial t}\left(\frac{\phi S_{\alpha}}{B_{\alpha}}\right)+\nabla \cdot\left(\frac{\mathbf{v}_{\alpha}}{B_{\alpha}}\right)-\frac{q_{\alpha, s}}{B_{\alpha}}=0$ for $\quad \alpha=w, o$

$\frac{\partial}{\partial t}\left[\phi\left(\frac{S_{g}}{B_{g}}+\frac{R_{s} S_{o}}{B_{o}}\right)\right]+\nabla \cdot\left(\frac{\mathbf{v}_{g}}{B_{g}}+\frac{R_{s} \mathbf{v}_{o}}{B_{o}}\right)$ $-\left(\frac{q_{g, s}}{B_{g}}+\frac{R_{s} q_{o, s}}{B_{o}}\right)=0$

$\mathbf{v}_{\alpha}=-\frac{k_{r \alpha} \mathbf{k}}{\mu_{\alpha}}\left(\nabla p_{\alpha}-\rho_{\alpha} \wp \nabla z\right) \quad$ for $\quad \alpha=w, o, g$

where indices $w, g$ and $o$ respectively denote water, oil and gas phases; $\mathbf{k}\left[\mathrm{L}^{2}\right]$ is the absolute permeability tensor; $\phi[-]$ is porosity; $\wp\left[\mathrm{LT}^{-2}\right]$ is gravity; $z[\mathrm{~L}]$ indicates a vertical coordinate; $t[\mathrm{~T}]$ is time; $R_{s}[-]$ is the dissolved gas-oil ratio; $q_{\alpha, s}\left[\mathrm{~T}^{-1}\right]$ is the source/sink term of phase $\alpha$ evaluated at standard conditions; $S_{\alpha}[-], p_{\alpha}\left[\mathrm{MT}^{-2} \mathrm{~L}^{-1}\right], \mu_{\alpha}\left[\mathrm{MT}^{-1} \mathrm{~L}^{-1}\right], \rho_{\alpha}\left[\mathrm{ML}^{-3}\right]$, $k_{r \alpha}\left[\mathrm{L}^{2}\right]$, and $\mathbf{v}_{\alpha}\left[\mathrm{LT}^{-1}\right]$ respectively denote saturation, pressure, viscosity, density, relative permeability and Darcy velocity of the $\alpha$-phase at the reservoir conditions; $B_{\alpha}[-]$ is the formation volume factor. Density $\rho_{\alpha}$ in (3) is evaluated as

$\rho_{w}=\frac{\rho_{w, s}}{B_{w}} ; \rho_{o}=\frac{R_{s} \rho_{g, s}+\rho_{o, s}}{B_{0}} ; \rho_{g}=\frac{\rho_{g, s}}{B_{g}}$

Here, $\rho_{\alpha, s}$ is the density of the $\alpha$-phase at standard conditions. Neglecting effects of the capillary pressure, solution of equations (1)-(4) is subject to (a) the constraint $S_{w}+S_{o}+S_{g}=1,(b)$ imposed initial and boundary conditions, as well as $(c)$ a set of mathematical formulations conducive to evaluating $k_{r \alpha}$.

Caudle et al. (1951) were among the first to highlight the impact of hysteresis effects (hereinafter denoted as HEs) on the values of relative permeability of a non-wetting phase. Occurrence of such effects for the non-wetting and the intermediate wetting phases have been documented by a set of experimental (e.g., Oak, 1990; Kalaydjian et al., 1997; Suicmez et al., 2007; Alizadeh and Piri, 2014a; Chukwudeme et al., 2014; Moghadasi et al., 2016) and theoretical (e.g., Killough, 1976; Carlson, 1981; Larsen and Skauge, 1998; Blunt, 2000; Shahverdi and Sohrabi, 2013; Kianinejad et al., 2015; Ranaee et al., 2015, 2016; Khorsandi et al., 2018) studies. A complete characterization of HEs for three-phase flow systems is still challenging due to complex pore-scale mechanisms driving fluid displacement. The main reasons underpinning the lack of reversibility of the saturation paths observed under three-phase conditions are (i) trapping of the non-wetting phase during imbibition, (ii) remobilization of the intermediate phase through a layer drainage displacement mechanism, and (iii) wettability alteration (change) during drainage and imbibition (Vizika and Lombard, 1996; Fenwick and Blunt, 1998; Van Dijke and Sorbie, 2003; Piri and Blunt, 2005; Van Dijke et al., 2006; Suicmez et al., 2007; Sohrabi et al., 2008; Blunt, 2017).

Available numerical tools usually employ empirical formulations to model three-phase relative permeabilities (e.g., Schlumberger Geo-Quest, 2010). Black-oil reservoir simulators commonly use laboratory- based two-phase (water-oil, gas-oil and/or water-gas) relative permeabilities as prior information to compute the spatial and temporal evolution of $k_{r \alpha}$ to be then embedded in (3). When HEs are neglected, the two-phase data can be inferred either from drainage or imbibition experiments. Otherwise, data from sets of two-phase drainage and imbibition experiments should be considered to evaluate $k_{r \alpha}$ (e.g., Killough, 1976; Carlson, 1981; Jerauld, 1997; Larsen and Skauge, 1998; Blunt, 2000; Lomeland and Ebeltoft, 2013; Ranee et al., 2015). Land (1968) developed a model to evaluate the amount of trapped gas during an imbibition process. Killough (1976) and Carlson (1981) proposed two formulations to evaluate HEs displayed by the non-wetting (i.e., gas in their setting) phase in a two-phase system. These models have been shown to generally underestimate the strength of HEs in three-phase environments (Larsen and Skauge, 1998; Spiteri and Juanes, 2006). Blunt (2000) introduced a relatively complex saturation-weighted interpolation procedure to include HEs, miscibility, composition and wettability change in the evaluation of $k_{r \alpha}$. This model imbues the impact of (i) layer drainage remobilization of the oil, and (ii) composition and miscibility of the hydrocarbon phases on oil relative permeability. Khorsandi et al. (2017) employed a compositional modeling framework to consider the impact of fluid compositions on phase relative permeabilities. A review of models that are commonly used to include HEs on gas relative permeability and their applicability in reservoir simulations is offered by Hoseini et al. (2011).

Here, we tackle three key questions: (i) Are black-oil reservoir simulations significantly affected by HEs exhibited by the relative permeability of the non-wetting and/or intermediate wetting phases? (ii) How does the impact of HEs vary with the initial spatial distribution of phase saturations? and (iii) Are HEs relevant in complex heterogeneous reservoir models? We analyze benefits and drawbacks of four strategies (which we term $S_{I}, S_{I I}, S_{I I I}$, and $S_{I V}$ ) that we consider to model $k_{r \alpha}$ under WAG injection protocols. All strategies are described in Section 2. The first two strategies are already implemented in commercial black-oil reservoir simulators (Schlumberger Geo-Quest, 2010) and either neglect HEs $\left(S_{I}\right)$ or consider HEs only for the gas phase $\left(S_{I I}\right)$. Strategy $S_{I I I}$ allows considering HEs solely for the oil phase and is grounded on the use of a model recently developed by Ranaee et al. (2015). HEs of both non- and intermediate-wetting phases are included in Strategy $S_{I V}$.

\section{Methodology}

Classical three-phase gas relative permeability $\left(k_{r g}\right)$ models (e.g., Killough, 1976; Carlson, 1981; Larsen and Skauge, 1998) allow including HEs by updating model parameters at a given location in the reservoir according to changes of the saturation path from drainage (i.e., increase of the non-wetting phase) to imbibition (i.e., increase of the wetting phase), or from imbibition to drainage. However, Suicmez et al. (2007) highlighted that solely relying on the identification of local drainage and imbibition conditions does not necessarily provide an exhaustive description of HEs on relative permeabilities under three-phase conditions because of the action of additional effects which might arise from changes of saturation paths.

In order to clarify this issue, here and in the following we illustrate our findings upon considering a water-wet medium. Making use of the nomenclature introduced by Snell (1962), the set of all possible saturation paths in a two- (flowing) phase system can be summarized by the following six conditions, termed as DIC, DCI, IDC, ICD, CDI, and CID. Here, first, second and third letters of each code respectively correspond to water, oil and gas, $\mathrm{C}$ is the non-flowing phase, I and D respectively refer to increasing and decreasing of phase saturation, bold letters specifying the non-wetting phase. Therefore, under two-phase conditions, drainage is described by the saturation paths DIC, DCI and CDI, while imbibition is characterized as IDC, ICD and CID. In other words, in two- (flowing) phase environments a description based on 
the concepts of drainage and imbibition cover all possible changes of saturation paths. Some models (e.g., Killough, 1976; Carlson, 1981) can include HEs on the evaluation of the relative permeability of the non-wetting phase due to possible switches of the saturation path (from drainage to imbibition conditions).

In the presence of three (flowing) phases, the six possible saturation paths of D I, DDI, IDD, DID and IID are not completely defined solely thro in terms of drainage and imbibition because these processes do not include any information about the displacement of the intermediate-wetting phase. In this context, it can be noted that saturation of both wetting and non-wetting phases can simultaneously increase (e.g., IDI) or decrease (e.g., DID).

Hysteresis effects on water can be neglected under water-wet conditions, as documented by experimental evidences (Oak, 1990; Di Carlo et al., 2000; Alizadeh and Piri, 2014b; Moghadasi et al., 2016) and theoretical analyses (Piri and Blunt, 2005; Spiteri and Juanes, 2006; Van Dijke et al., 2006; Suicmez et al., 2008; Bianchi Janetti et al., 2015). Accordingly, we can set three-phase water relative permeabilities $\left(k_{r w}\right)$ to the values associated with their counterparts observed during either drainage or imbibition in a water-oil, $\bar{k}_{r w o}$, or water-gas, $\bar{k}_{r w g}$, system. For this reason, we neglect in the following HEs on $k_{r w}$ due to changes of saturation path from DDI to IDI (and vice versa) as well as from DID to IID (and vice versa). Oil and gas relative permeabilities (respectively denoted as $k_{r o}$ and $k_{r g}$ ) are evaluated according to the strategies detailed in the following subsections and summarized in Table 1.

\subsection{Strategy $I\left(S_{I}\right)$}

Hysteresis effects on oil and gas relative permeabilities are neglected in this setting. Therefore, this modeling strategy can be used when changes of gas and oil saturation path are negligible across the simulation domain and the temporal window of interest. Three-phase gas relative permeability, $k_{r g}$, is set equal to its two-phase counterpart, $\bar{k}_{r g o}^{d}$, evaluated during drainage in a gas-oil environment (Lie, 2014).

Various models are available to evaluate three-phase oil relative permeability, $k_{r o}$. Commercial black-oil simulators (e.g., Schlumberger Geo-Quest, 2010) typically consider $k_{r o}$ as the result of a saturation-weighted interpolation (Baker, 1988) and/or through the Stone (1970, 1973) models. Ranaee et al. (2016) show that Stone-type models lead to loss of hyperbolic flow behavior (see also Juanes and Patzek, 2004; and Bianchi Janetti et al., 2015 for detailed analyses on this condition) in some ranges of fluid saturations under three-phase conditions. As such, these models do not capture the detailed physics of the three-phase flow in porous systems for some saturation scenarios. Following these observations, $k_{r o}$, is evaluated according to the non-hysteretic (saturation-weighted interpolation) model (Baker, 1988)

$k_{r o}=\frac{\left(S_{w}-\bar{S}_{w c}\right) \bar{k}_{r o w}^{i}+S_{g} \bar{k}_{r o g}^{d}}{S_{w}-\bar{S}_{w c}+S_{g}}$

Table 1

Models used for the evaluation of intermediate- and non-wetting phase relative permeabilities through strategies $S_{I}$ to $S_{I V}$, embedding Hysteretic Effects (HEs) as detailed in Section 2 .

\begin{tabular}{lllll}
\hline Strategies & $k_{r o}$ & $k_{r g}$ & $\begin{array}{l}\text { HEs on } k_{r o}(\mathrm{Y}: \mathrm{Yes} ; \mathrm{N}: \\
\mathrm{No})\end{array}$ & $\begin{array}{l}\text { HEs on } k_{r g}(\mathrm{Y}: \mathrm{Yes} ; \mathrm{N}: \\
\text { No) }\end{array}$ \\
\hline$S_{I}$ & $(5)$ & $\bar{k}_{r g o}^{d}$ & $\mathrm{~N}$ & $\mathrm{~N}$ \\
$S_{I I}$ & $(5)$ & $(6)-(9)$ & $\mathrm{N}$ & $\mathrm{Y}$ \\
$S_{I I I}$ & $(10)-(14)$ & $\bar{k}_{r g o}^{d}$ & $\mathrm{Y}$ & $\mathrm{N}$ \\
$S_{I V}$ & $(10)-(14)$ & $(6)-(9)$ & $\mathrm{Y}$ & $\mathrm{Y}$ \\
\hline
\end{tabular}

Here, $\bar{S}_{w c}$ is connate water saturation in a water-oil system and $\bar{k}_{\text {row }}^{i}$ is oil relative permeability evaluated during imbibition in an oil-water system, all remaining symbols being already defined.

\subsection{Strategy II $\left(S_{I I}\right)$}

Here, HEs are considered only for the non-wetting phase (i.e., gas in our exemplary setting). Therefore, $S_{\text {II }}$ does not capture HEs on the oil phase due to possible changes of saturation paths between IDI and DII as well as between IDD and IID. This modeling strategy is commonly adopted in commercial black-oil reservoir simulators and can be used when changes of saturation paths for the oil phase are negligible.

A variety of models have been developed to account for HEs on gas relative permeability under three-phase scenarios (Lenhard and Parker, 1987; Lenhard and Oostrom, 1998; Larsen and Skauge, 1998; Egermann et al., 2000). Spiteri and Juanes (2006) show that the model proposed by Larsen and Skauge (1998) should be selected under WAG injection protocols. According to this formulation, gas relative permeability during imbibition, $k_{r g}=k_{r g}^{I}$, and drainage, $k_{r g}=k_{r g}^{D}$, are respectively evaluated as

$$
\begin{aligned}
k_{r g}^{I}\left(S_{g}\right)= & \bar{k}_{r g o}^{d}\left(S_{g f}\right) ; k_{r g}^{D} \\
= & k_{r g}^{I}\left(S_{g i}\right) \\
& +\left(\bar{k}_{r g o}^{d}\left(S_{g}\right)-\bar{k}_{r g o}^{d}\left(S_{g i}\right)\right)\left(\frac{\bar{S}_{w c}}{S_{w i}}\right)^{C_{\alpha}}
\end{aligned}
$$

Here, $C_{\alpha}$ is the secondary drainage reduction coefficient, $S_{g i}$ and $S_{w i}$ respectively are gas and water saturations at the beginning of gas drainage, and $S_{g f}$ is flowing gas saturation given by

$$
\begin{array}{r}
S_{g f}=\bar{S}_{g c}+\frac{1}{2}\left\{S_{g}-S_{g t}\right. \\
\left.+\sqrt{\left(S_{g}-S_{g t}\right)\left(S_{g}-S_{g t}+\frac{4}{C_{L}}\right)}\right\}
\end{array}
$$

where $\bar{S}_{g c}$ is the critical gas saturation corresponding to the onset of gas-oil drainage (see Fig. 1a), $C_{L}$ is the Land trapping coefficient, and $S_{g t}$ is the trapped gas saturation for a three-phase system at the end of an imbibition process. The latter is computed as (Land, 1968)

$$
\begin{aligned}
S_{g t} & =\bar{S}_{g c}+\frac{S_{g i}-\bar{S}_{g c}}{1+C_{L}\left(S_{g i}-\bar{S}_{g c}\right)} \text { with } C \\
& =\left(\bar{S}_{g t}-\bar{S}_{g c}\right)^{-1}-\left(\bar{S}_{g o}^{M}-\bar{S}_{g c}\right)^{-1}
\end{aligned}
$$

Here, $\bar{S}_{g o}^{M}$ is the largest gas saturation observed in an oil-gas system at the end of gas injection; and $\bar{S}_{g t}$ is trapped gas at the end of oil injection (see also Fig. 1a). Note that according to (6), and following the depiction in Fig. 1a, gas relative permeability values obtained during gas injection (i.e., drainage) are slightly larger in two-phase oil-gas environments than in three-phase systems. According to Blunt (2000), the total trapped hydrocarbon (as expressed in terms of oil and gas saturations, i.e., $S_{g t}+S_{o r}$ ) in a three-phase (water-wet) environment under imbibition is approximately equal to the residual oil saturation in a two-phase oil-water setting, $\bar{S}_{\text {row }}$ (see Fig. 1c). Therefore, an alternative formulation to evaluate $S_{g t}$ can be

$S_{\text {gt }}=\bar{S}_{\text {row }}-S_{\text {orem }}$ with $S_{\text {orem }}<\bar{S}_{\text {row }}$

$S_{\text {orem }}$ representing remaining oil saturation, i.e., the fraction of pore volume occupied by oil, at a given time under three-phase conditions. 

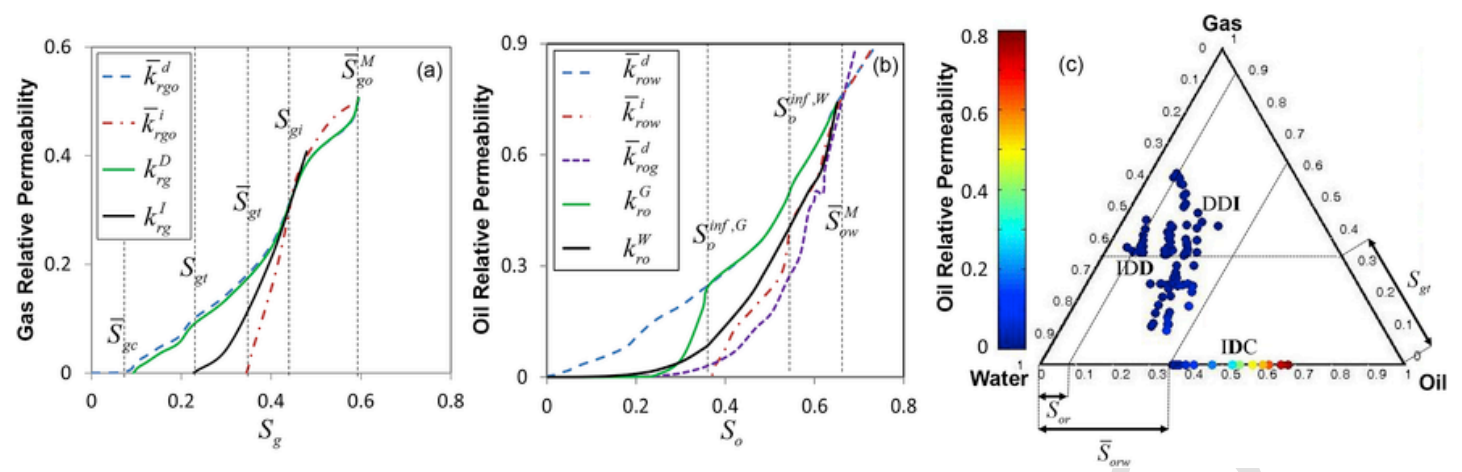

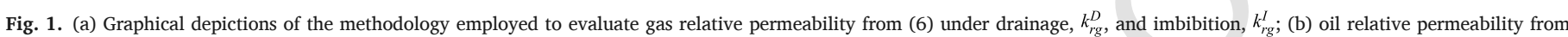

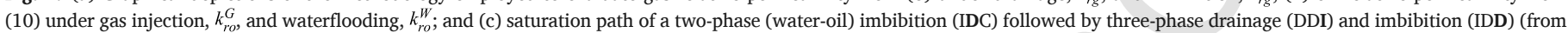
Oak, 1990).

\subsection{Strategy III $\left(S_{I I I}\right)$}

We provide here a methodology conducive to including HEs on the evaluation of oil relative permeability while neglecting HEs on gas relative permeability, the latter being set as $k_{r g}=\bar{k}_{r g o}^{d}$ as in strategy $S_{I}$. This approach should be consistent during SWAG procedures, when water and gas are simultaneously injected to displace oil and/or during primary waterfloding, i.e., when the change in gas content is mainly corresponding to decreasing gas saturations.

Ranaee et al. (2015) introduced the following sigmoid-based (SB) model to capture HEs in the evaluation of three-phase oil relative permeability under waterflooding, $k_{r o}=k_{r o}^{W}$, and gas injection, $k_{r o}=k_{r o}^{G}$ (see Fig. 1b),

$$
\begin{aligned}
k_{r o}^{W} & =\frac{\left(S_{w}-\bar{S}_{w c}\right) k_{r o}^{S, W}+\left(S_{g}-\bar{S}_{g t}\right) \bar{k}_{r o g}^{d}}{\left(S_{w}-\bar{S}_{w c}\right)+\left(S_{g}-\bar{S}_{g t}\right)} ; k_{r o}^{G} \\
& =\min \left[\bar{k}_{r o w}^{d}, \max \left(k_{r o}^{S, G}, \bar{k}_{r o g}^{d}\right)\right] .
\end{aligned}
$$

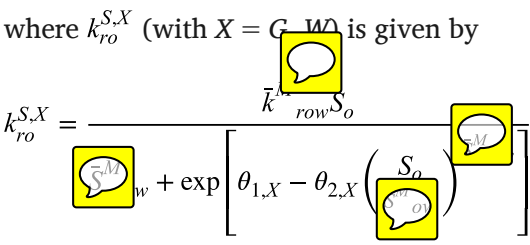

Here, $\bar{k}_{\text {row }}^{M}$ is the value of $\bar{k}_{\text {row }}^{i}$ corresponding to oil saturation $\bar{S}_{o w}^{M}=1-\bar{S}_{w c}$. Model parameters $\theta_{1, X}$ and $\theta_{2, X}$ in (11) can be evaluated on the basis of two-phase data (oil-gas and oil-water) according to

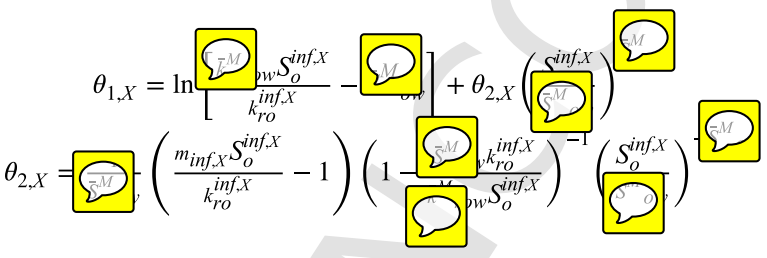

where

$$
\begin{aligned}
S_{o}^{\mathrm{inf}, G} & =S_{o i} ; S_{o}^{\mathrm{inf}, W} \\
& =\bar{S}_{r o w}+\frac{\bar{S}_{o w}^{M}-\bar{S}_{r o w}}{2} ; k_{r o}^{i n f, G} \\
& =\bar{k}_{r o w}^{d}\left(S_{o}^{i n f, G}\right) ; k_{r o}^{i n f, W} \\
& =\bar{k}_{\text {row }}^{i}\left(S_{o}^{i n f, W}\right)
\end{aligned}
$$

$m_{i n f, G}=\left.\frac{\partial \bar{k}_{r o g}^{d}}{\partial S_{o}}\right|_{\bar{S}_{w c}} ; m_{i n f, W}=\left.\frac{\partial \bar{k}_{r o w}^{i}}{\partial S_{o}}\right|_{S_{o}^{i n f}}$

$S_{o i}$ in (13) being initial oil saturation evaluating onset of the switches of saturation paths from waterflooding to gas injection conditions (all remaining symbols having already been defined).

Ranaee et al. (2015, 2016; 2017) demonstrated the ability of (10)-(14) to interpret coreflooding $k_{r o}$ data (Oak, 1990; Di Carlo et al., 2000; Alizadeh and Piri, 2014b; Moghadasi et al., 2016) collected under diverse saturation histories and wettability conditions.

\subsection{Strategy $I V\left(S_{I V}\right)$}

Here, HEs for both oil and gas relative permeabilities are considered, via (10)-(14) and (6)-(9), respectively. Ranaee et al. (2018a) illustrated the details of the numerical implementation of this strategy for reservoir simulation applications.

A summary of the main features of all of the above illustrated strategies is given in Table 1.

\section{Results}

In the following we analyze the impact of HEs on reservoir simulation outputs considering a relatively simple stratified reservoir (Section 3.1) and a complex heterogeneous system (Section 3.2). In order to include strategies $S_{I T} S_{I V}$ in our analysis we extend the MRST toolbox (Lie, 2014), originally developed to implement only $S_{I}$. During reservoir simulation, we evaluate and update relative permeability values of each of the numerical blocks forming the system according to their saturation history and available two-phase relative permeability data deduced from Oak (1990) and shown in Fig. 2. The following two-phase saturation characteristic values are evaluated from Fig. 2: connate water, $\bar{S}_{w c}=0.275$; trapped gas, $\bar{S}_{g t}=0.345$; residual oil from water-oil and gas-oil environments respectively being set as $\bar{S}_{\text {orw }}=0.365$ and $\bar{S}_{\text {org }}$ $=0.13$.

\subsection{Homogeneous reservoir model}

Here, we consider a two-dimensional reservoir model for the implementation of a WAG injection scenario in the presence of a single producer and a single injection well. The computational domain is depicted in Fig. 3. The reservoir has a size of $10000 \times 10000 \mathrm{ft}$ in the horizontal plane. The domain is formed by $10 \times 10$ cells of uniform size along the horizontal plane and thickness equal to $50 \mathrm{ft}$. Porosity and absolute permeability are constant and respectively set equal to 0.3 and $200 \mathrm{mD}$ as indicated in Fig. 3. 

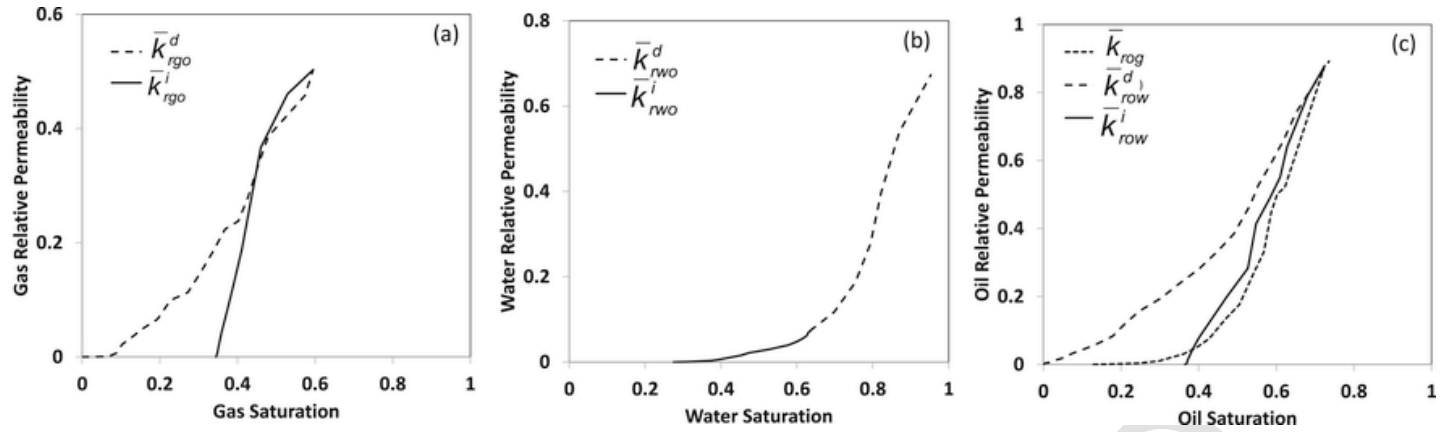

Fig. 2. Two-phase relative permeability data from Oak (1990), employed for the characterization of the reservoir simulation models.

(a)

(b)

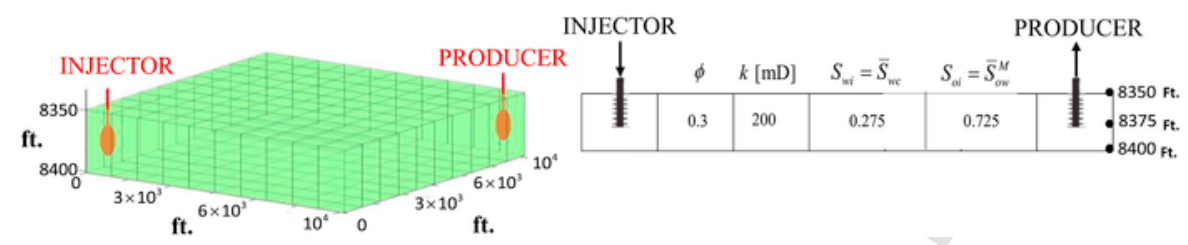

Fig. 3. Homogeneous reservoir model: (a) global view, and (b) diagonal cross-section and input data.

The reservoir is initially saturated with oil at two-phase connate water, i.e., $S_{o i}=1-\bar{S}_{w c}, S_{w i}=\bar{S}_{w c}$, and $S_{g i}=0$. The initial pressure distribution is hydrostatic, with a pressure value $p=4800$ psia at the center of the reservoir (corresponding to vertical location $z=8375 \mathrm{ft}$ ). Densities of water, oil and gas at standard conditions are set equal to 64.80 , 49.10 and $60.54 \times 10^{-3} \mathrm{lb} / \mathrm{ft}^{3}$, respectively. Table 2 lists Pressure-Volume-Temperature (PVT) properties of the reservoir live oil and dry gas, while PVT properties of water are set as

$$
B_{w}(p)=\frac{B_{w, r e f}}{1+C_{w}\left(p-P_{r e f}\right)\left(1+C_{w}\left(p-P_{r e f}\right) / 2\right)}
$$

where $B_{w, r e f}=1.03 \mathrm{rb} / \mathrm{stb}, \mu_{w}=0.31 \mathrm{cP}$, and $C_{w}=3.13 \times 10^{-6} 1 / \mathrm{psi} \mathrm{re-}$ spectively are the formation volume factor, viscosity and compressibility of water at the reference pressure $P_{\text {ref }}=4014.7$ psia.

Table 2

PVT conditions for oil and gas. $P_{b u b}$ represents bubble point pressures.

\begin{tabular}{llll}
\hline \multicolumn{2}{l}{ PVT conditions of oil } & & \\
\hline$P_{b u b}(\mathrm{psia})$ & $R_{s}(\mathrm{Mscf} / \mathrm{stb})$ & $B_{o}(\mathrm{rb} / \mathrm{stb})$ & $\mu_{o}(\mathrm{cP})$ \\
\hline 14.7 & 0.001 & 1.062 & 1.04 \\
264.7 & 0.0905 & 1.15 & 0.975 \\
514.7 & 0.18 & 1.207 & 0.91 \\
1014.7 & 0.371 & 1.295 & 0.83 \\
2014.7 & 0.636 & 1.435 & 0.695 \\
2514.7 & 0.775 & 1.5 & 0.641 \\
3014.7 & 0.93 & 1.565 & 0.594 \\
4014.7 & 1.27 & 1.695 & 0.51 \\
5014.7 & 1.618 & 1.827 & 0.449 \\
\hline
\end{tabular}

PVT conditions of gas

\begin{tabular}{lll}
\hline$P(\mathrm{psia})$ & $B_{g}(\mathrm{rb} / \mathrm{Mscf})$ & $\mu_{g}(\mathrm{cP})$ \\
\hline 14.7 & 166.67 & 0.008 \\
264.7 & 12.09 & 0.0096 \\
514.7 & 6.274 & 0.0112 \\
1014.7 & 3.197 & 0.014 \\
2014.7 & 1.614 & 0.0189 \\
2514.7 & 1.294 & 0.0208 \\
3014.7 & 1.08 & 0.0228 \\
4014.7 & 0.811 & 0.0268 \\
5014.7 & 0.649 & 0.0309 \\
\hline
\end{tabular}

Injector and producer wells have a diameter equal to $0.5 \mathrm{ft}$ and are located at two opposite corners of the domain (see Fig. 3). The producer is controlled by imposing a 4790 psia bottom-hole pressure (BHP).

A WAG injection generally aims at combining high macroscopic sweep efficiency of the waterflooding operation with high displacement efficiency of the gas injection process to improve oil recovery (Kulkarni and Rao, 2005). Performances of WAG projects are typically governed by the number of WAG cycles, the fluid volume of each cycle, and the injection rates of the gas and water phases (Speight, 2009). Optimal values of such operational parameters can depend on reservoir host rock and fluid characteristics. Here, we analyze the effects of these WAG control parameters on the reservoir simulation responses to obtain an indication about optimum conditions in terms of (i) the time at which the WAG procedure can be initiated following primary waterflooding, (ii) injection rates, and (iii) the duration of each WAG cycle to ensure efficiency of the gas/water mobility ratios.

We start by computing the ultimate oil recovery, UOR, defined as the field oil efficiency (FOE) evaluated at the total production life time, i.e.,

$$
\begin{aligned}
& \mathrm{UOR}=\mathrm{C}\left(t_{L C}\right) ; \text { with } \mathrm{FOE}(t) \\
& =\frac{\sum_{j=1} \sum_{i=1}^{n_{t}} \bar{q}_{o, j}^{i} \Delta t_{i}}{\text { FOIIP }}
\end{aligned}
$$

Here, $n_{P}$ is the number of production wells, $\Delta t_{i}$ is the selected time step, $n_{t}$ is the number of time steps, $\bar{q}_{o, j}^{i}$ is the oil production rate associated with the $j$-th production well at the $i$-th time step, and FOIIP $=$ $S_{o i} \phi V / B_{o i}$ represents initial oil in place for a reservoir of total volume $V$. In order to include economic aspects in the evaluation of the well control efficiencies, we also evaluate the net present value, NPV (Chen and Reynolds, 2015), definas

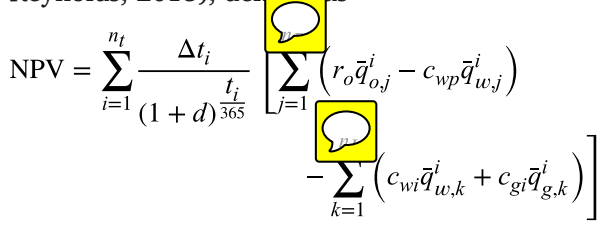

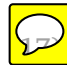

where $n_{I}$ denotes the number of injection wells, $\bar{q}_{\alpha, j}^{i}$ and $\bar{q}_{\alpha, k}^{i}$ respec- 
tively are the production (when $\alpha=w, o$ ) and injection (when $\alpha=w, g$ ) rate of the $\alpha$-phase at the $i$-th time step, $d$ is the annual discount (inflation) rate, $r_{o}$ is oil revenue; $c_{w p}, c_{w i}$ and $c_{g i}$ respectively being the disposal cost of produced water, injected water and injected gas. For illustration purposes and following Chen and Reynolds (2015), we set $d=0.1, r_{o}=80 \$ / \mathrm{stb}, c_{w p}=5 \$ / \mathrm{stb}, c_{w i}=5 \$ / \mathrm{stb}$, and $c_{g i}=1.5 \$ /$ Mscf.

The WAG injection scheme is scheduled according to the following procedure. We start by a primary waterflooding (denoted as W1); we then alternate gas injection and waterflooding (each WAG cycle being characterized by the same duration $\left(t_{C Y C}\right)$ and being structured according to gas injection during half of the time frame, followed by waterflooding) until a total production life $t_{L C}=50$ years. Fayers et al. (1991) show the most efficient displacement of the WAG injection can be obtained when both water and gas phases move in the reservoir at equal pore velocities. We then set the injector well operational control mode to inject a constant pore volume (PV) throughout the full extent of the simulation $\left(t_{L C}\right)$ and switch the injected phase from water to gas (and vice versa) during primary waterflooding and WAG cycles.

Fig. 4 depicts the dependence of net-present-value (NPV) on (dimensionless) duration of primary waterflooding, $t_{W 1} / t_{L C}$, and (dimensionless) duration of each WAG cycle, $t_{C Y C} / t_{L C}$. Results in Fig. 4 are given for three differing values of injection rates (expressed in terms of pore volumes, i.e., $\mathrm{PV}=1,2$, and 5 ) and for all simulation strategies
( $S_{I}$ to $S_{I V}$ ) described in Section 2. Hereinafter we denote as "base case" the test case where only $W 1$ is implemented, i.e., $t_{W 1}=t_{L C}$.

Our numerical simulations suggest that WAG procedures yield a higher NPV than does the base case only if the injection rate is sufficiently large (in our case, $P V \geq 2$ ). These results indicate that NPV strongly depends on the duration of $W 1$ for medium $(P V=2)$ to high $(P V=5)$ injection rates, being otherwise insensitive to it for low $(\mathrm{PV}=1)$ injection rate. When $t_{W 1} / t_{L C}$ is sufficiently large (i.e., $t_{W 1} / t_{L C}>0.7$ in our case) and WAG is implemented, we note that the inclusion of HEs on the evaluation of relative permeabilities does not affect the evaluation of NPV. With the increase of the injection rate (from $\mathrm{PV}=2$ to $\mathrm{PV}=5$ ), NPV is largest for the lower $t_{W 1} / t_{L C}$. Hence, NPV does not change significantly with $t_{C Y C} / t_{L C}$ in the considered range, an exception being given by the results in Fig. $4 \mathrm{f}$. When the injection rate is set to $\mathrm{PV}=2$ and HEs on oil relative permeability are taken into account (i.e., $S_{I I I}$ and $S_{I V}$ ), NPV values are characterized by a very sharp peak localized at $t_{W 1} / t_{L C} \cong 0.2$. Otherwise (i.e., $S_{I}$ and $S_{I I}$ ), the NPV values remain approximately constant across the range $0.1 \leq t_{W 1} / t_{L C} \leq 0.5$, being always lower than their counterparts associated with $S_{I I I}$ and $S_{I V}$. Inclusion of HEs in the evaluation of gas relative permeability (i.e., $S_{I I}$ ) does not impact significantly NPV as compared against the results obtained via $S_{I}$.

Fig. 5 depicts $S_{g t}$ evaluated from (8) versus the corresponding values computed from (9). It can be observed that both equations provide similar results when $S_{g t}$ is smaller than approximately 0.1 . Otherwise,

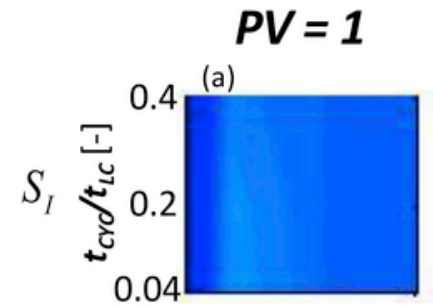

(d)
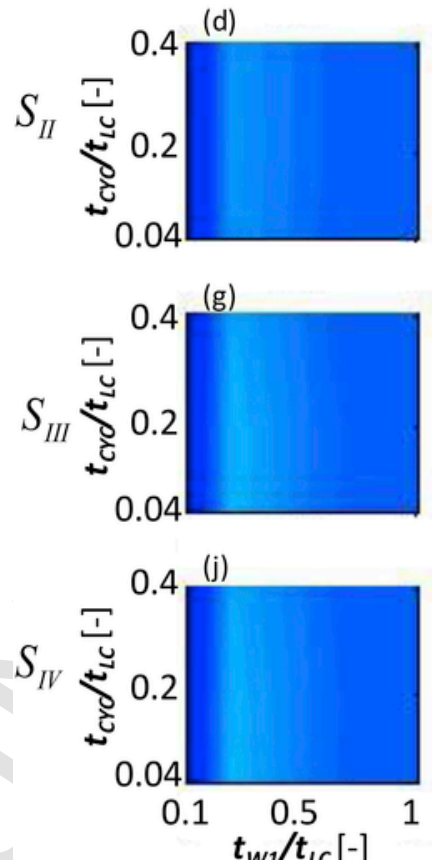

$P V=2$

(b)

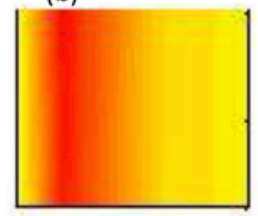

(e)

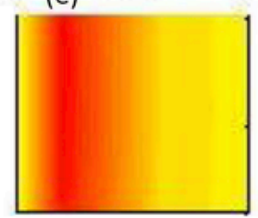

(h)

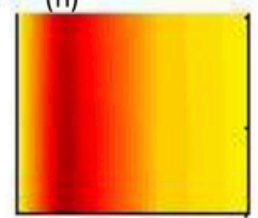

(k)

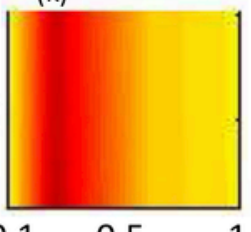

$0.1 \quad 0.5 \quad 1$
$P V=5$

(c)

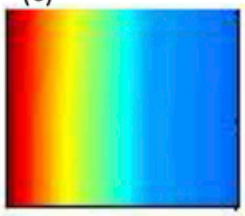

(f)

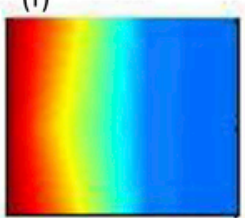

(i)

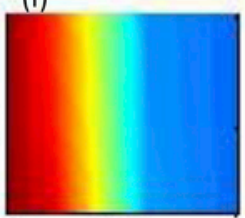

(I)

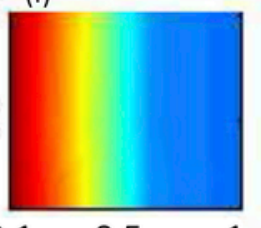

$0.1 \underset{t_{w 1} / t_{L C}[-]}{0.5} 1$ $\times 10^{7}$

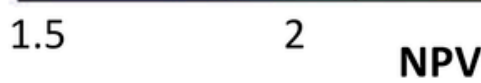

2.5

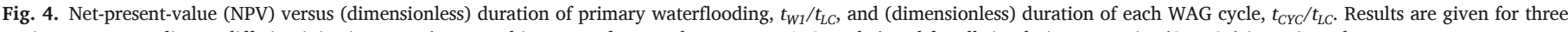
settings, corresponding to differing injection rates (expressed in terms of pore volumes, $\mathrm{PV}=1,2$, and 5 ) and for all simulation strategies $\left(S_{I}\right.$ to $\left.S_{I V}\right)$ investigated. 


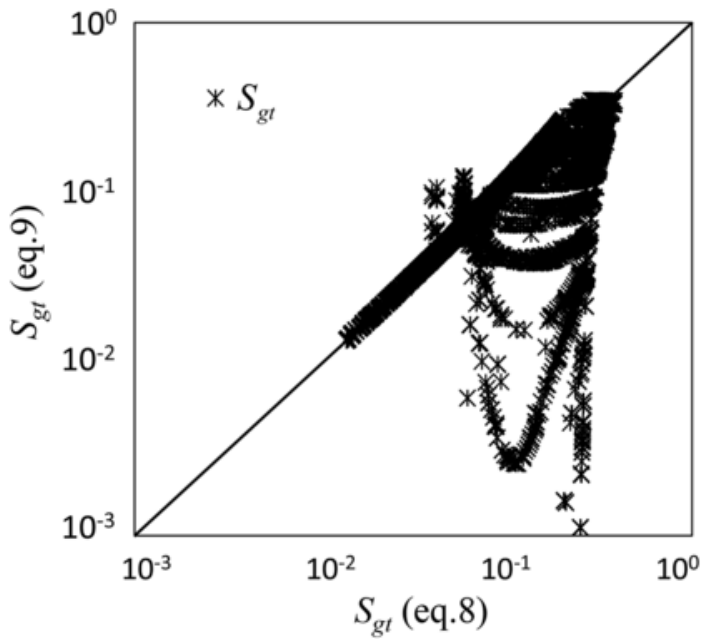

Fig. 5. Trapped gas saturation, $S_{g t}$ evaluated from (9) versus its counterpart computed via (8) in the context of strategy $S_{I V}$. Results are rendered when primary waterflooding is set at $t_{W 1} / t_{L C}=0.1$.

i.e., for $S_{g t}>0.1$, (9) yields values of $S_{g t}$ significantly smaller than those evaluated through (8). Therefore, one can conclude that reliance on either of the two formulations may affect $S_{I I}$ and $S_{I V}$ only in the presence of quite large values of $S_{g t}$.

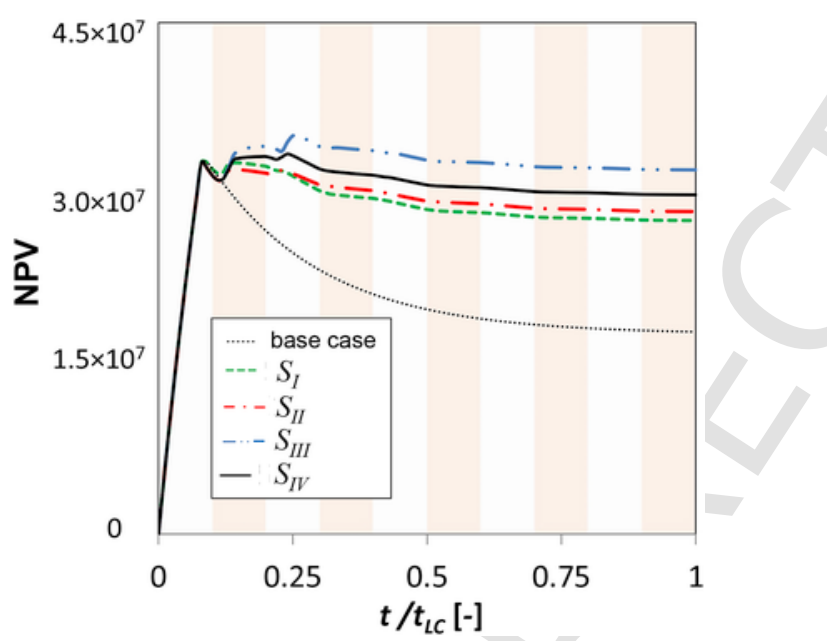

Fig. 6. Temporal evolution of NPV for production under a WAG protocol designed with $t_{W 1} / t_{L C}=0.1, t_{C Y C} / t_{L C}=0.2$, and PV $=5$. Background colors correspond to gas (pink background) and water (white background) injection phases. (For interpretation of the references to color in this figure legend, the reader is referred to the Web version of this article.)
Fig. 6 depicts NPV versus the dimensionless duration of the simulation time $\left(t / t_{L C}\right)$ obtained for all simulation strategies investigated. Quantities illustrated in Figs. 6-9 have been evaluated upon setting $t_{W 1} / t_{L C}=0.1, t_{C Y C} / t_{L C}=0.2$ and PV $=5$ to clearly investigate the effects of relative permeability models on evaluation of NPV. In this case, values of $S_{g t}$ are smaller than 0.1 , so that (8) and (9) render very similar results. As such, $S_{g t}$ has been evaluated via (8) in the following, as usually performed in existing commercial reservoir simulators.

Results of Fig. 6 show that the highest increase of NPV from the base case is associated with $S_{I I I}$, $S_{I}$ being characterized by the lowest difference from the base case. Note that results from $S_{I I}$ are very similar to those of $S_{I}$. Fig. 6 suggests that inclusion of HEs in the evaluation of gas relative permeability does not influence the evaluation of NPV as much as embedding oil relative permeability hysteresis.

Fig. 7 depicts (a) field water-cut, FWCT $=Q_{w} /\left(Q_{0}+Q_{w}\right)$, (b) FOE and (c) produced field gas-oil ratio (FGOR $=Q_{g} / Q_{o}$ ) versus $t / t_{L C}, Q_{\alpha}$ being the sum of the producti tes of fluid phase $\alpha$ across all $n_{p}$ producing wells, i.e., $Q_{\alpha}=\sum_{i=1}^{n_{t}} \sum_{j=1} \bar{q}_{\alpha, j}^{i}$. Results show that all curves overlap for $t / t_{L C}<0.1$, while production is under the sole action of waterflooding. The WAG procedure starts with gas injection at time $t / t_{L C}=$ 0.1 and all models lead to a decrease of FWCT and an increase of FOE and FGOR as compared to the base case. All modeling strategies yield similar results in terms of FWCT which oscillates in time, water breakthrough being attained at times longer than those associated with the base case. We note that relying on the non-hysteretic model $S_{I}$ yields the lowest increase of FOE as compared to the base case. Inclusion of HEs in the description of only $k_{r o}$ (i.e., strategy $S_{\text {III }}$ ) yields the highest values of FOE and the lowest values of FGOR. Otherwise, considering HEs solely for $k_{r g}\left(S_{I I}\right)(a)$ leads to some increase of FOE as compared against $S_{I}$ and (b) yields the highest increase of FGOR from the base case. Compared to $S_{I I I}$, inclusion of HEs in the evaluation of both $k_{r o}$ and $k_{r g}$ (i.e., $S_{I V}$ ) yields FOE and FGOR values in between those resulting from $S_{I I}$ and $S_{I I I}$ (when HEs are neglected either on $k_{r o}$ or $k_{r g}$, respectively).

Pore-scale analyses of multiphase displacement mechanisms can provide some insights to support results of the kind documented in Fig. 7. Dong et al. (2005) focus on micro-model simulations and report that disconnected oil clusters can be mobilized in a multiple-displacement setting (of the kind that take place under WAG conditions), most of those clusters being essentially immobile during primary waterflooding. Zhang et al. (2006) evidence that pore-scale fluid displacement mechanisms during WAG in a water-wet environment are chiefly piston-like, with the possibility of oil snap-off during primary waterflooding. The gas injected into the system can invade pores previously filled by immobilized oil (due to the reduced gas/oil interfacial tension, IFT, as compared to water/oil conditions). A stable oil layer then tends to form between the gas and water phases. Consequently, the (pore-scale)
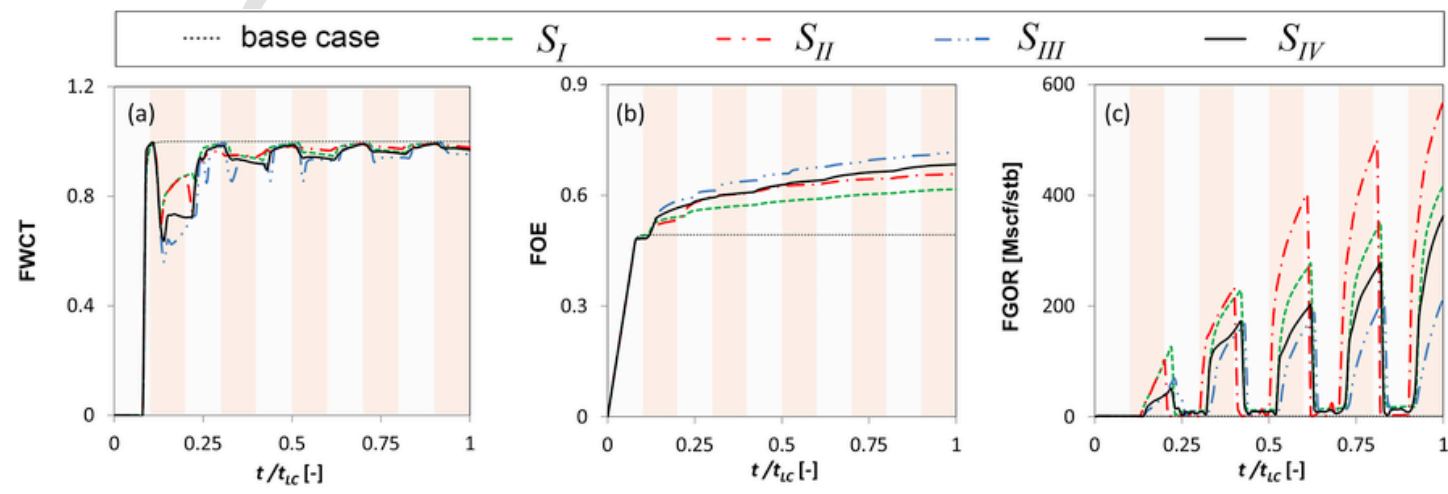

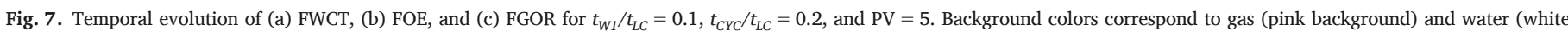
background) injection phases. (For interpretation of the references to color in this figure legend, the reader is referred to the Web version of this article.) 
(a)

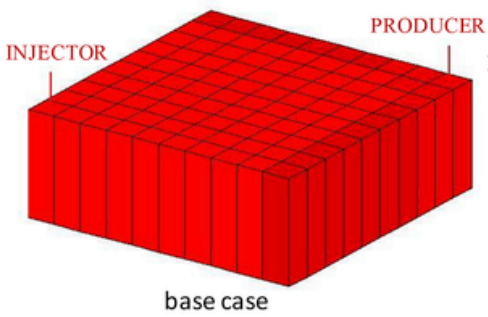

(d) (b)

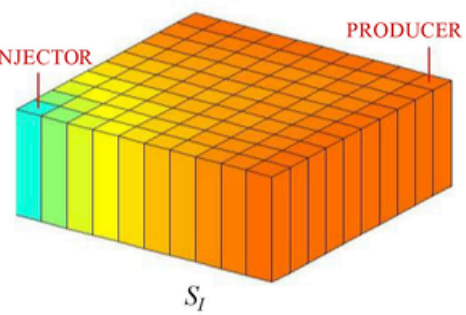

(e)

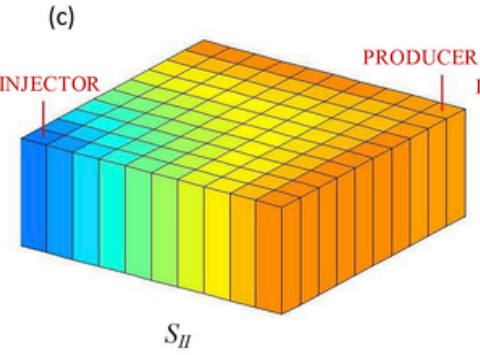

$S_{I I}$

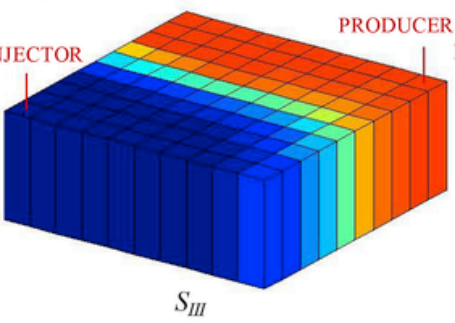

PRODUCER

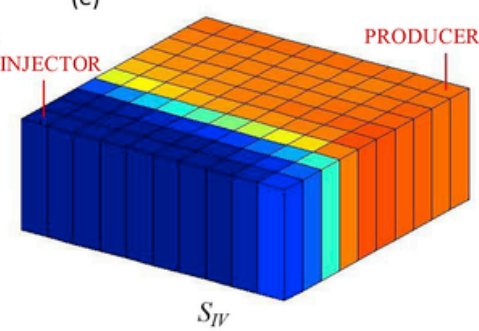

0.1

0.2

$S_{\mathrm{o}}$

0.3

0.4

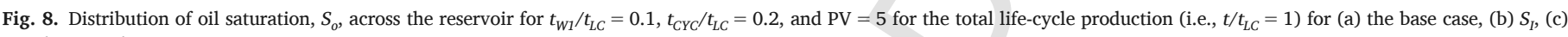
$S_{I I}$, (d) $S_{I I}$, and (e) $S_{I V}$.
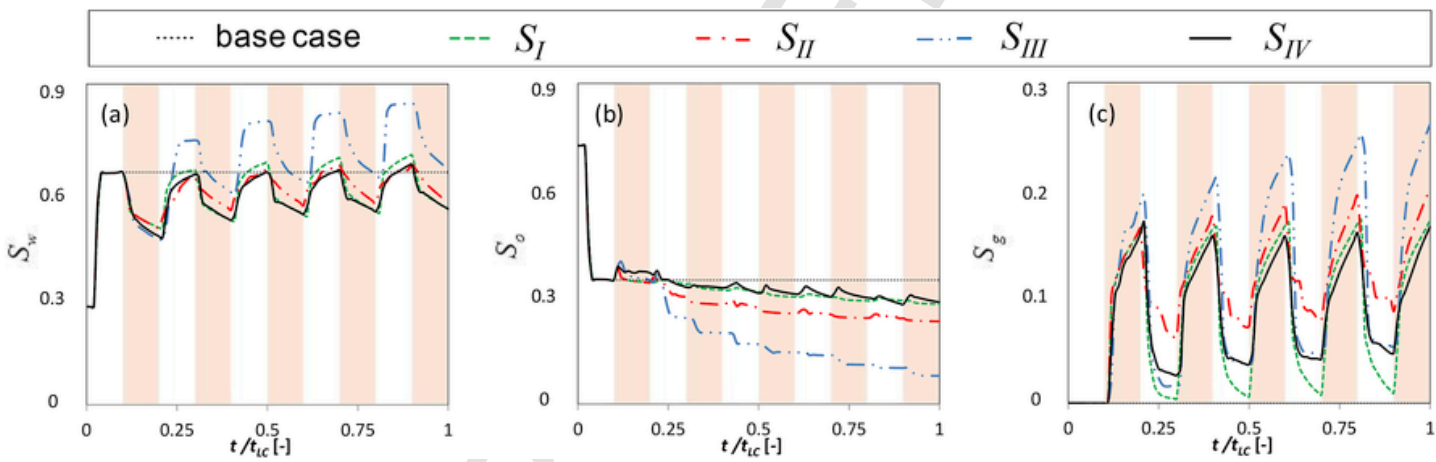

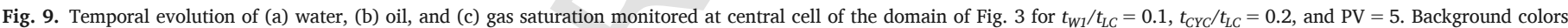

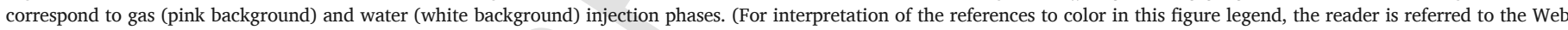
version of this article.)

displacement under WAG cycles tends to be associated with a layer drainage behavior rather than being governed by a piston-like mechanism (Suicmez et al., 2007; Piri and Blunt, 2005; Van Dijke et al., 2002). In this context, it can be noted that layer drainage remobilization of oil can lead to gas trapping, a feature that is consistent with the decrease of FGOR in our results of considering HEs (see Fig. 7c). Note that the amount of trapped gas is a strong function of the IFT between the oil and gas, a feature strengthening the value of further investigation on IFT impacts on gas trapping during WAG (Afzali et al., 2018).

Due to blockage effects of gas bubbles (Dong et al., 2005), the oil/ water blobs are not displaced in some pores during the first WAG injection cycle. Gas bubbles may snap-off at the pore throats and become discontinuous during the following water injection cycles due to the interplay of capillary forces and local gas pressure fluctuations (Sohrabi et al., 2004). Additional oil can then be recovered in the next WAG cycles. This is mainly due to two mechanisms: (i) water displacing the oil that was trapped in water invaded pores (during primary waterflooding and/or previous WAG cycles), and (ii) water displacing the oil that was trapped in other regions when the gas refilled the water-occupied channels. These observations are in line with the increase of recovery for subsequent WAG cycles illustrated in Fig. 7b for the models considering HEs of the oil phase (i.e., $S_{\text {III }}$ and $S_{I V}$ ).

Further to these observations, Sohrabi et al. (2004) show that relative permeability reduction and injectivity loss may occur during subsequent WAG cycles due to gas trapping. Cyclic injection of water and gas leads to fragmentation and trapping of gas in pores and throats across the pore space. As a consequence, the area available to flow is limited and oil and gas relative permeability for the subsequent WAG cycles is reduced, as compared to the first WAG cycle. This mechanism is consistent with the milder increase of oil recovery and gas-oil ratio documented in Fig. 7 for the subsequent WAG cycles associated with the models considering HEs of the oil phase (i.e., $S_{I I I}$ and $S_{I V}$ ).

Fig. 8 depicts the distribution of oil saturation $\left(S_{o}\right)$ across the reservoir at the end of the life-cycle production time (i.e., at $t / t_{L C}=1$ ) for the base case (Fig. 8a) and following the implementation of WAG procedures for the differing modeling strategies considered (Fig. 8b-e). When compared against the base case, the results illustrate the least decrease of $S_{o}$ during WAG for $S_{I}-S_{I I}$. Otherwise, a clear reduction of $S_{o}$ is observed when HEs on the evaluation of $k_{r o}$ are considered $\left(S_{I I I}\right.$ and $\left.S_{I V}\right)$. 
Fig. 8 clearly show that oil saturation, $S_{o}$, for $S_{I I I}$ and $S_{I V}$ can tend to very small values in some parts of the domain. We note that typical applications tend to assume that $\bar{S}_{\text {org }}<S_{\text {or }}<\bar{S}_{\text {orw }}$ (e.g., Fayers and Matthews, 1984) to evaluate three-phase residual oil saturation. This can be supported by considering that gas-oil IFT is lower than water-oil IFT, thus resulting in an enhanced quantity of oil being displaced from the pore spaces due to gas injection as compared to what can be obtained through primary waterflooding (Afzali et al., 2018). Secondary gas injection can then lead to changes of relative permeability curves as well as residual oil saturation, increasing oil recovery.

Recent observations and laboratory data confirm that $S_{\text {or }}$ can take values smaller than $\bar{S}_{\text {org }}$ (Oak, 1990; Alizadeh and Piri, 2014b; Kianinejad and DiCarlo, 2016; Moghadasi et al., 2016) and, in some cases, $S_{o r}$ can even tend to zero (e.g., Blunt, 2000; Jerauld, 1997 and references therein). Wettability alteration might occur during WAG practices due to various factors including, e.g., asphaltene precipitation, water salinity, or $\mathrm{CO}_{2}$ effects (Abdi et al., 2014; Patel et al., 1987). Moreover, one can note that, as opposed to results from strictly immiscible WAG simulations, miscibility effects between gas and oil phases might take place during typical WAG practices. The IFT value associated with miscible scenarios can tend to vanish. This results in a high value of the capillary number, favoring values of three-phase residual oil saturation in the swept region that can be lower than $\bar{S}_{\text {org }}$ (Henderson et al., 1998) or even zero (Shahverdi et al., 2011). The Sigmoid model embeds all these features, enabling $S_{\text {or }}$ to diminish and to eventually attain negligible values.

During the primary water flooding process of our test case, oil recovery is increasing with constant rate because of the piston-like displacement of the injected water that governs oil sweep. As such, the displacement front tends to be perpendicular to the diagonal from injector to producer. High rates of gas injection (e.g., PV $=5$ for the test case depicted in Fig. 8) during WAG are associated with a high mobility difference between the displacing and displaced phase. The latter can in turn lead to an increase of the possibility of viscosity fingering phenomena to take place and cause instabilities of the displacement front (Virnovsky et al., 1994; Berg and Ott, 2012). The occurrence of such instabilities is supported by pore-scale observations (see, e.g., Sohrabi et al., 2004) that reveal the fluids in each cycle of the WAG injection process tend to find new flow pathways in the porous medium. These processes can then lead to asymmetry of the displacement front and saturation distribution even through an otherwise homogeneous reservoir (see also Adebayo et al., 2017).

Evaluation of $k_{r o}$ through non-hysteresis saturation-weighted interpolation model (e.g., $S_{I}$ and $S_{I I}$ ) leads to almost vanishing oil mobility ratio for the subsequent WAG cycles, thus hampering the possibility to represent instability of the oil displacement front (due to high gas in-

(a)

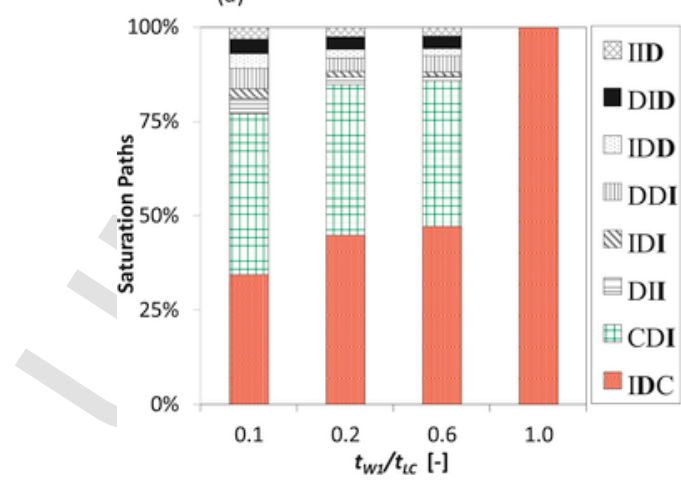

jection rate). Otherwise, including HEs on our evaluations of $k_{r o}$ leads to some local remobilization of oil and nonzero values of oil flowing ratio. These, in turn, give rise to a non-uniform expansion of the displacement front for the subsequent WAG cycles. This feature can be clearly recognized from the steep displacement front of the oil saturation depicted in Fig. 8d and e for $S_{I I I}$ and $S_{I V}$.

The significance of the impact of hysteresis on $k_{r o}$ on the reduction of water, oil and gas saturations is strengthened by the results of Fig. 9 where the saturation paths obtained in the central block cell are depicted for the setting corresponding to $t_{W 1} / t_{L C}=0.1, t_{C Y C} / t_{L C}=0.2$, and $\mathrm{PV}=5$. These results show that employing strategies $S_{I}, S_{I I}$ and $S_{I V}$ lead to a value of residual oil saturation, $S_{\text {or }}$, slightly smaller than $\bar{S}_{\text {orw }}$ (i.e., 0.365 ) while $S_{I I I}$ yields a value of $S_{\text {or }}$ significantly smaller than $\bar{S}_{\text {org }}$. Model $S_{\text {III }}$ provides the highest increase of $S_{w}$ and $S_{g}$ from the base case, during waterflooding and gas injection cycles, respectively. Meanwhile, the non-hysteresis model $\left(S_{I}\right)$ yields to the lowest values of $S_{g}$ during gas injection cycles.

Oscillations observed in the saturation histories of Fig. 9 support the occurrence of changes of saturation paths during the simulations. Fig. 10 depicts the relative fraction of occurrences of two- and three-phase saturation paths across all computational cells and the complete temporal window spanned by our simulations as a result of the implementation of strategy $S_{I V}$ for five differing values of $t_{W 1} / t_{L C}$ when $t_{W 1} / t_{L C}$ is fixed to 0.2. Similar results (not shown) have been documented in the presence of strategies $S_{\Gamma} S_{I I I}$. When the injection rate is set to $\mathrm{PV}=5$ (Fig. 10a), the percentage of the observed saturation paths corresponding to three-phase conditions increases with the decrease of primary waterflooding duration. This suggests that NPV values tend to increase with decreasing $t_{W 1}$ for high injection rates. Comparing results of Figs. $10 \mathrm{~b}$ and $\mathrm{a}$ we note that decreasing the injection rate (from PV $=5$ to $\mathrm{PV}=2$ ) leads to $(i)$ a reduction of the region where three-phase flow take place for all values of $t_{W 1} / t_{L C}$ and (ii) an increase of the duration $t_{W 1} / t_{L C}$ for which one can observe that the fraction of the domain occupied by three-phase flow conditions is largest, i.e., the value of $t_{W 1} / t_{L C}$ for which the highest percentage of the saturation paths belong to three-phase environment (e.g., $t_{W 1} / t_{L C}=0.1$, or 0.2 in Fig. $10 \mathrm{a}$ and b, respectively). When $t_{W 1}$ is larger than its optimum value (corresponding to $\left.t_{W 1} / t_{L C}=0.2\right)$ for $\mathrm{PV}=2$, the volumetric fraction of regions where three-phase flow takes place decreases and simulation results document a poor efficiency of WAG procedures in terms of NPV (see also Fig. 4).

Considering Fig. 4 for PV $=5$ in conjunction with the results depicted in Fig. 10a, one can conclude that WAG can be inefficient to improve NPV for $t_{W 1} / t_{L C}>0.5$ compared to a base case of waterflooding at very high injection rates, even if three-phase regions are observed through the simulation domain. These results are supported by previ-

(b)

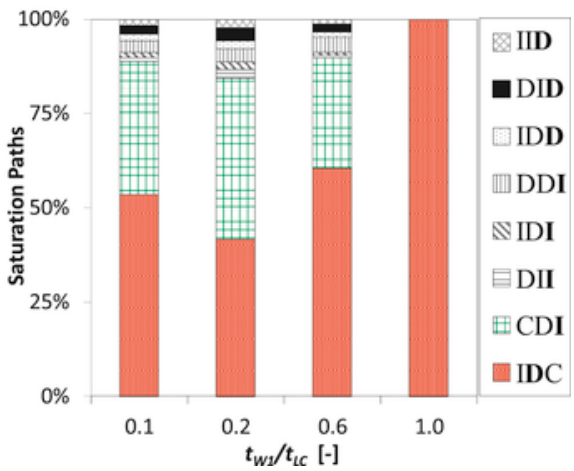

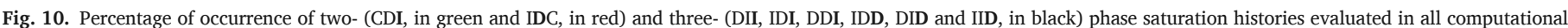

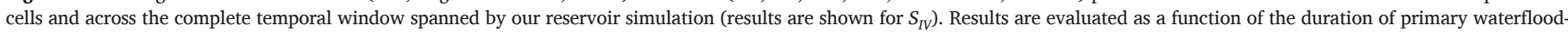
ing, $t_{W 1} / t_{L C}$, for $t_{C Y C} / t_{L C}=0.2$ and (a) $\mathrm{PV}=5$, (b) $\mathrm{PV}=2$. (For interpretation of the references to color in this figure legend, the reader is referred to the Web version of this article.) 
ous observations (Christensen et al., 2001) which show that viscous instabilities can happen under high WAG injection rates and can make a WAG practice inefficient. Note that the efficiency of a WAG protocol can be even more influenced by the injection rate for a heterogeneous reservoir model (Afzali et al., 2018) while the flow rate in each reservoir region is proportional to the local flow capacity (i.e., the local conductivity). Then, injectivity problems may also occur under high injection rates due to: (i) different horizontal/vertical connectivity, permeability and porosity properties and/or (ii) high permeability along fractures.

\subsection{Effects of complexity of reservoir model properties}

In some cases, failures of WAG projects have been attributed to the spatial heterogeneity of the reservoir properties (e.g., Afzali et al., 2018 and references therein).

Here, we analyze the impact of HEs on oil and gas relative permeabilities in a complex heterogeneous reservoir model with multiple injection and production wells. To this end, we select the PUNQ-S3 model (Floris et al., 2001), which is a well-known reservoir model originally developed as a test case for production forecasting under uncertainty. The reservoir is bounded by sealing faults along all sides and is composed by fluvial sand and shale. The top of the reservoir is at a depth of $7677 \mathrm{ft}$. The average reservoir thickness is $92 \mathrm{ft}$. The reservoir is discretized into $19 \times 28 \times 5$ non-uniform grid blocks. A map of the spatial distribution of absolute horizontal (i.e., $k_{x}=k_{y}$ ) permeability and porosity values is depicted in Fig. 11. Average porosity is 0.2 , and the average horizontal permeability is $100 \mathrm{mD}$. The vertical permeability, $k_{z}$, is set as $k_{y} / 3$ (Floris et al., 2001). The reservoir pore volume is almost 200 million barrels with initial oil saturation of about $72.5 \%$. The initial reservoir pressure is set to 3670 psia. The model has four injection wells and four production wells (see Fig. 11). All wells are screened across layers $3-5$ of the reservoir.

We modify the original PUNQ-S3 model to include a WAG scenario. The original PUNQ-S3 model is characterized by a dome with a small gas cap in the center. Here, we remove the gas cap and, similar to the setting analyzed in Section 3.1, the entire reservoir is initially saturated with oil at two-phase connate water saturation, i.e., $S_{o i}=1-\bar{S}_{w c}$ , $S_{w i}=\bar{S}_{w c}, S_{g i}=0$. We then consider simulation scenarios where the production wells operate at a fixed BHP of 3670 psia. The injection rate of each of the injector wells INJ-j $(j=1, \ldots, 4)$ is set at $1885 \mathrm{stb} /$ day during waterflooding and at $1885 \mathrm{Mscf} /$ day during gas injection. We start with a primary waterflooding (denoted as W1) encompassing a temporal window of duration equal to $t_{W 1}=5$ years. We then alternate gas injection and waterflooding for the injectors on a 5-year basis up to a total production life, $t_{L C}$, of 50 years. Note when wells INJ-1 and INJ-2 are set for waterflooding, wells INJ-3 and INJ-4 are injecting gas, while INJ-3 and INJ-4 inject water whenever INJ-1 and INJ-2 are set to inject gas. All remaining reservoir properties coincide with those employed in Section 3.1.

WAG efficiency is evaluated by implementing (i) $S_{I}$, i.e., neglecting HEs on oil and gas, and (ii) $S_{I V}$, i.e., considering HEs on both the non-wetting and intermediate wetting phase.

Fig. 12 depicts the evolution of water, oil, and gas rates (respectively denoted as $\bar{q}_{w, j}, \bar{q}_{o, j}$, and $\bar{q}_{g, j}$ ) with (dimensionless) time, $t / t_{L C}$, at the producer well PRO-j $(j=1, \ldots, 4)$. The base case, where all injection wells are injecting water for the full production time, is also depicted.

It can be noted that even as one considers that the initial state of a reservoir is characterized by the absence of free gas, there can be significant amounts of gas dissolved in the oil phase. For a given pressure condition $(p)$, there is a fixed amount of gas that can be dissolved, according to (1)-(4). One can then note from Fig. 13 and Fig. 14 that free gas appears within the first simulation steps even for the base case of waterflooding.

As expected, $\bar{q}_{w, j}$ decreases and $\bar{q}_{g, j}$ increases under the WAG process as compared to the base case. Due to the heterogeneity of the domain, oil production rate increases at two of the producing locations (PRO-1 and PRO-3) while it is not affected by the enhanced oil recovery procedure employed (i.e., waterflooding or WAG) in PRO-2 and PRO-4. Producing rates of all fluid phases are only slightly affected by HEs. One can note that the combined action of HEs and of the heterogeneity of the field can cause an increase or a decrease of the production rates depending on the location considered.

Fig. 13 depicts (a) FWCT, (b) FOE and (c) FGOR versus $t / t_{L C}$. As expected, all modeling strategies compared provide similar trends during primary waterflooding $\left(0<t / t_{L C}<0.1\right)$, FWCT and FGOR respectively decreasing and increasing following WAG implementation, as compared to the base case. In this setting, we further note that inclusion of HEs in the modeling workflow yields results suggesting that WAG is effective in increasing FOE (with respect to the base case) at late production times. This observation is further supported by Fig. 14, where the spatial distribution of $S_{o}$ at $t / t_{L C}=1$ is depicted for (a) the base case and when WAG is implemented with (b) $S_{I}$ and (c) $S_{I V}$. A clear reduction of $S_{o}$ (as compared to the base case) is observed throughout the domain only when $S_{I V}$ is employed.

Finally, Fig. 15 depicts NPV versus $t / t_{L C}$. Application of WAG appears to promote an increased NPV as compared to the base case, independent of the inclusion of HEs in the modeling process. Indeed, the increase of NPV (with respect to the base case) observed for $S_{I}$ is not due to an enhancement of field oil production, being otherwise linked to a decrease of water production (see also Fig. 13). On the other hand, the
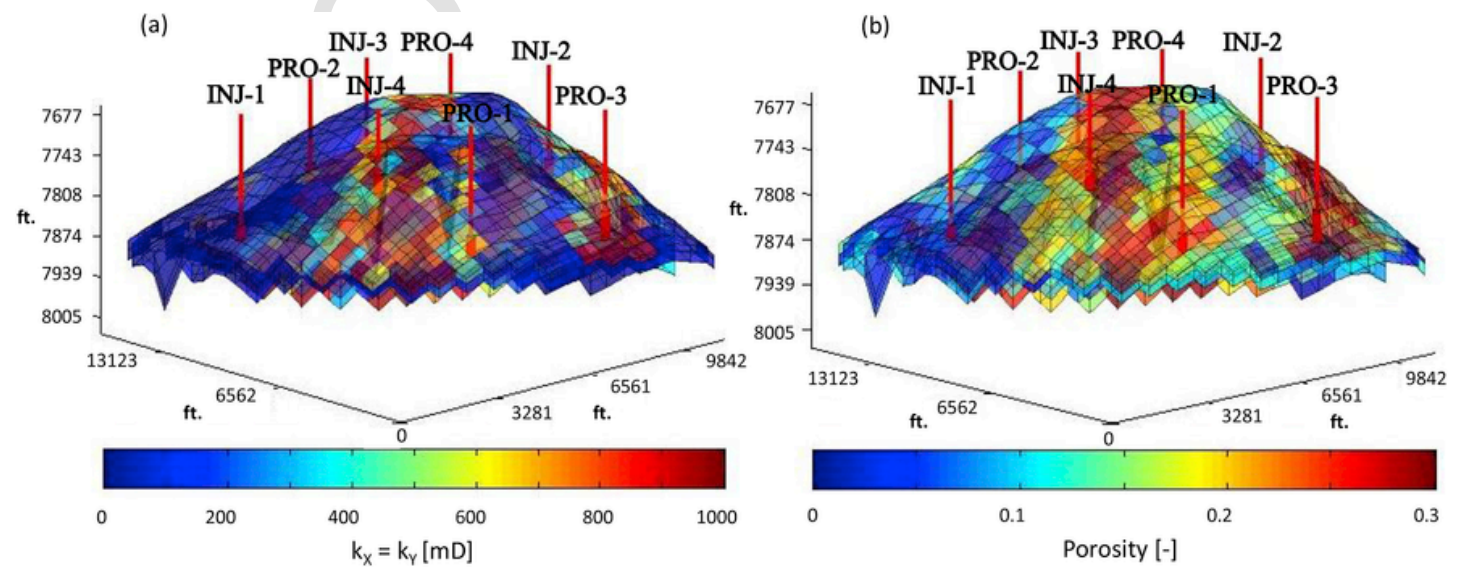

Fig. 11. Three-dimensional view of the PUNQ-S3 reservoir model with well locations and distribution of (a) absolute permeability and (b) porosity. 

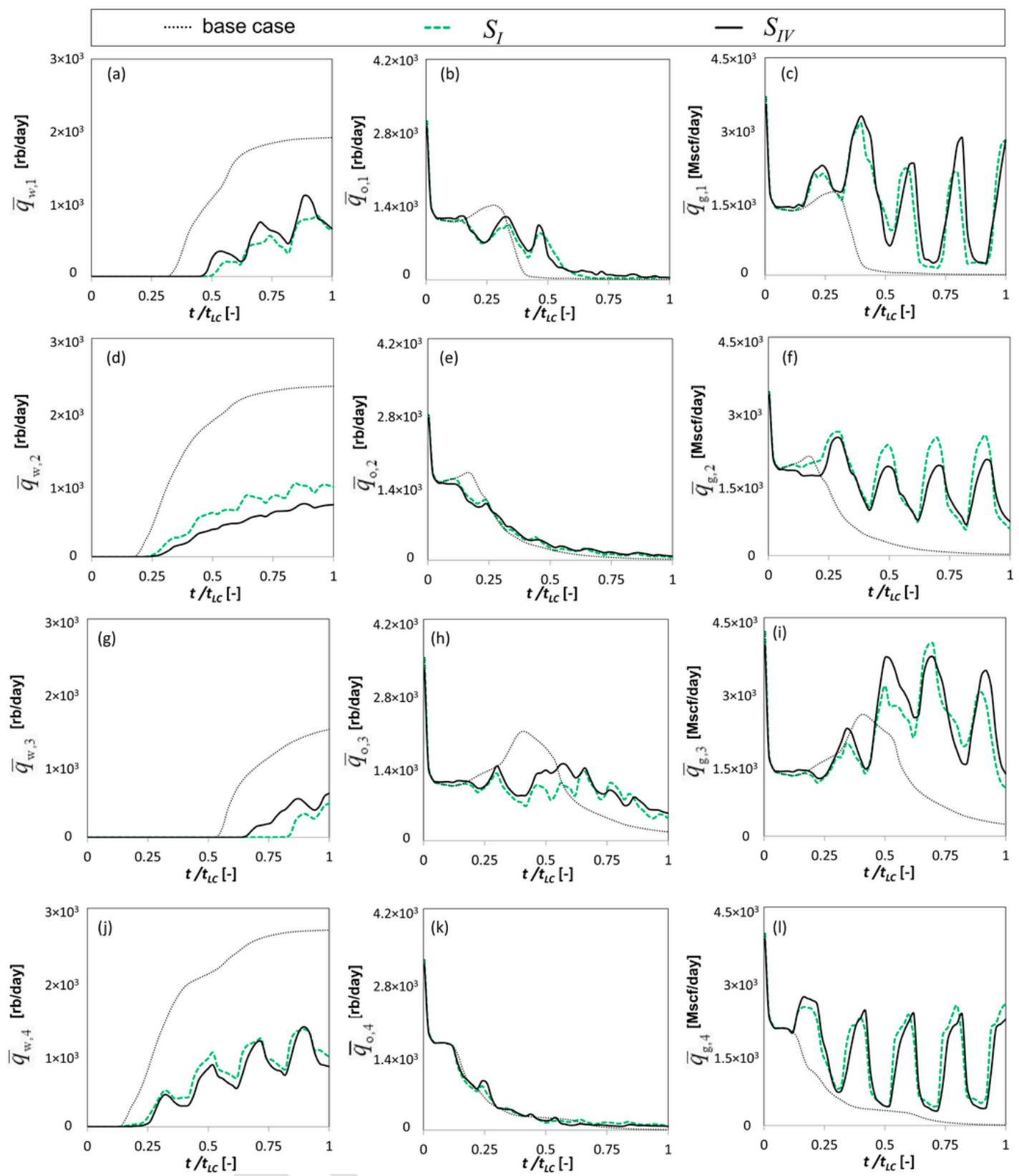

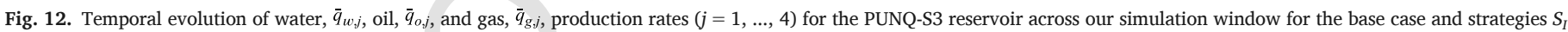
and $S_{I V}$.
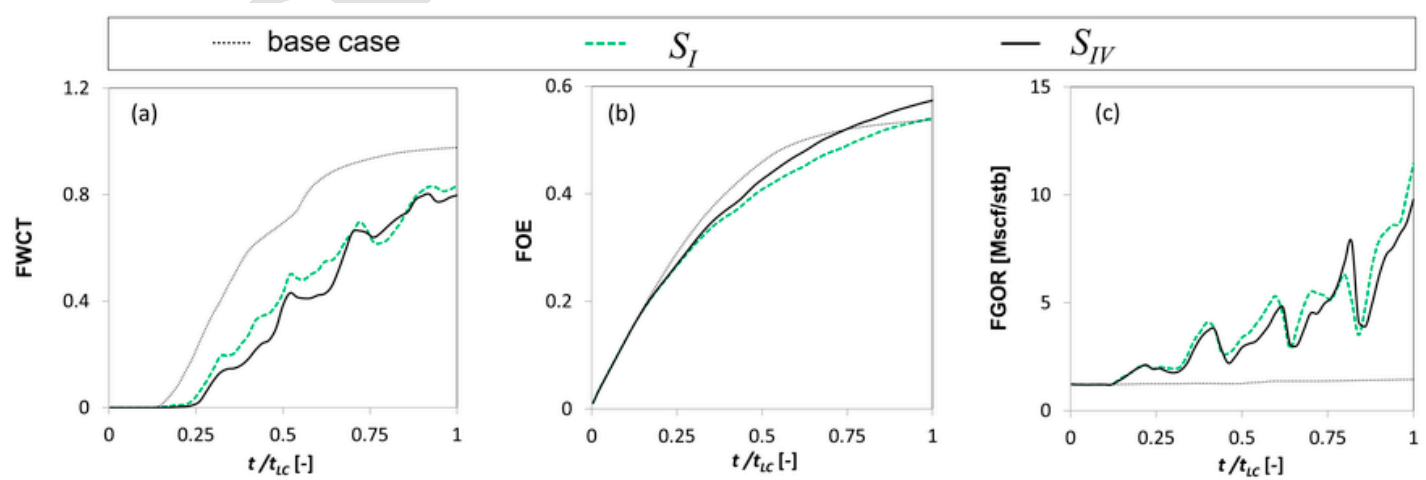

Fig. 13. Temporal evolution of (a) FWCT, (b) FOE and (c) FGOR for PUNQ-S3 reservoir across our simulation window for the base case and strategies $S_{I}$ and $S_{I V}$. 


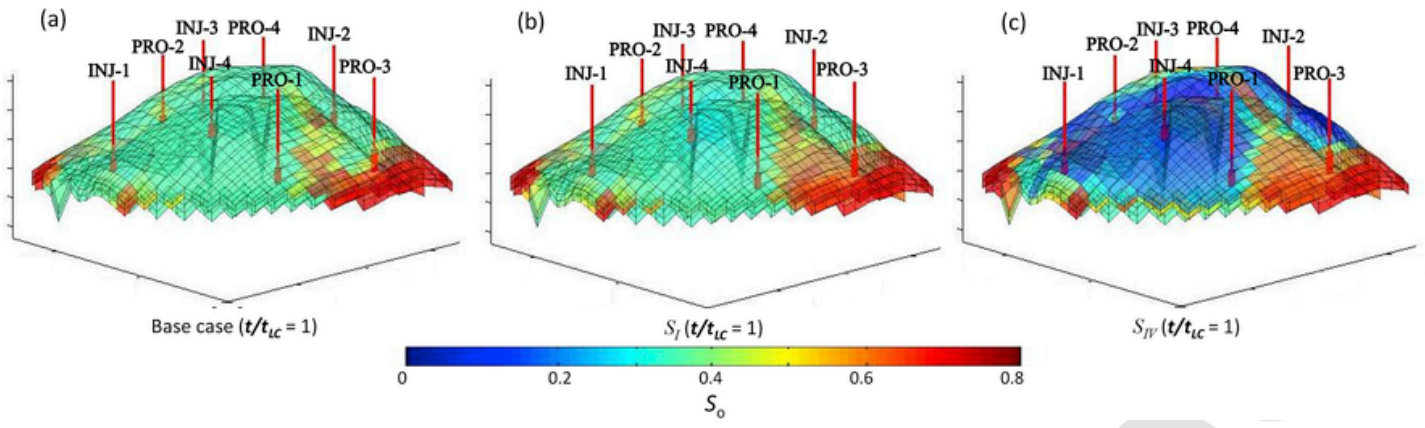

Fig. 14. Distribution of oil saturation, $S_{o}$, through the PUNQ-S3 reservoir domain at $t / t_{L C}=1$ for (a) the base case, and modeling strategies (b) $S_{I}$, (c) $S_{I V}$.

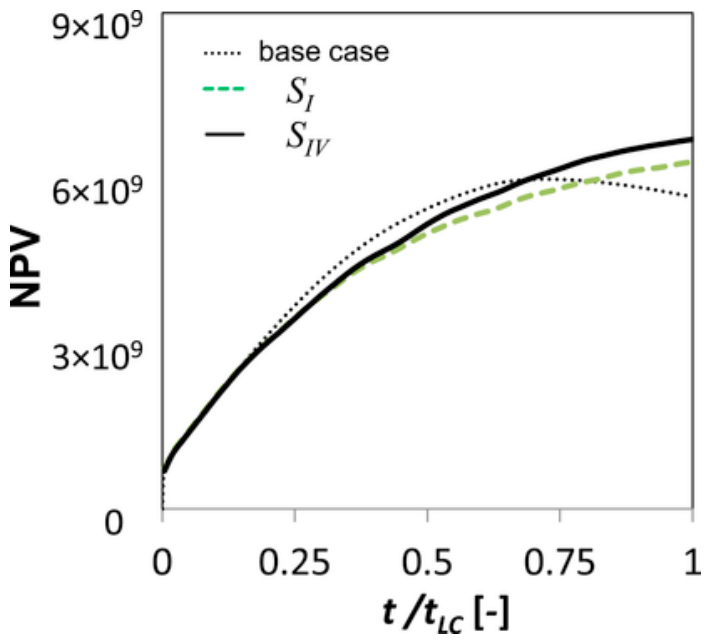

Fig. 15. Temporal evolution of NPV for the PUNQ-S3 reservoir resulting from simulations associated with the base case and $S_{I}$ and $S_{I V}$.

inclusion of HEs on the evaluation of relative permeabilities leads to a significant increase of NPV (up to about $17 \%$ at $t=t_{L C}$ ) with respect to the base case due to simultaneous increase of oil production and decrease of water production.

\section{Conclusions}

We study the impact of explicitly including HEs for relative permeabilities in numerical simulations of enhanced oil recovery approaches grounded on the application of WAG injection protocols. The analysis is performed in the context of black-oil simulation strategies and considering scenarios implemented in a simple homogeneous reservoir as well as in a complex heterogeneous domain. The latter is designed relying on the well-known PUNQ-S3 reservoir model, which is adapted to include a WAG scenario. Four strategies are analyzed to model relative permeabilities of fluid phases under WAG scenarios targeting water-wet reservoirs. Two of these are already implemented in commercial black-oil reservoir simulators and either neglect HEs (strategy $S_{I}$ ) or consider HEs only for the gas phase $\left(S_{I I}\right)$. Strategy $S_{I I I}$ considers HEs only for the oil phase while $S_{I V}$ considers HEs of both non- and intermediate-wetting phases. Impacts of the selection of each of these strategies are assessed through comparison against a base case where oil recovery is guided only through waterflooding. An assessment of the reservoir responses to the effects of the main WAG parameters is presented. Our study leads to the following major conclusions.

1. Efficiency of a WAG protocol is sensitive to the duration of primary waterflooding only if the injection rate is set to a sufficiently high value. Oil production does not change significantly with the (dimensionless) duration of the WAG cycles, $t_{C Y C} / t_{L C}$, in the range of values explored.

2. Our numerical results reveal that implementation of WAG can be effective in increasing FOE (field oil efficiency) and NPV (net present value) with respect to a base case, only when HEs in the evaluation of oil relative permeabilities are explicitly modeled. Otherwise, the inclusion of HEs for gas relative permeability does not impact significantly NPV.

3. Inclusion of HEs in the numerical model typically yield an increased FOE and a decreased FGOR (produced field gas-oil ratio), as compared to neglecting them. Our study also shows that the spatial distribution of fluid saturations at the onset of the WAG operation, as driven by the duration of primary waterflooding, can impact the values of FOE and FGOR. In this context, we show that significant portions of the reservoir can be characterized by saturation paths corresponding to three-phase flow conditions following an appropriate selection of the duration of primary waterflooding preceding WAG implementation.

4. As compared against an oil recovery approach based solely on waterflooding, numerical simulations of WAG embedding hysteresis on three-phase relative permeabilities yield an increased NPV due to a simultaneous significant increase of oil and decrease of water at the producers.

A preliminary and explorative appraisal of an increased complexity geological setting is illustrated in Ranaee et al. (2018b). These authors show that inclusion of HEs (as compared to neglecting them) in the numerical model can yield some delay in gas breakthrough, and consequently, an increased ultimate oil recovery and a mild decrease of gas-oil ratio and water cut, a finding that is consistent with the results of our current study.

\section{Acknowledgement}

This work was partially supported by a grant provided by Eni S.p.A. "Modellazione alla microscala e parametri effettivi per tecniche WAG". SINTEF Applied Mathematics is acknowledged for allowing the use of MRST for research purposes.

\section{Appendix A. Supplementary data}

Supplementary data to this article can be found online at https:// doi.org/10.1016/j.petrol.2019.01.044.

SI Metric Conversion Factors

\begin{tabular}{|c|c|}
\hline $\mathrm{rb} \times 1.589873$ & $=10^{-1} \mathrm{~m}^{3}$ \\
\hline $\mathrm{cP} \times 1.0$ & $=10^{-3} \mathrm{Pas}$ \\
\hline $\mathrm{ft} \times 3.048$ & $=10^{-1} \mathrm{~m}$ \\
\hline
\end{tabular}




\begin{tabular}{ll}
\hline $\mathrm{mD} \times 9.869233$ & $=10^{-4} \mu \mathrm{m}^{2}$ \\
$\mathrm{psi} \times 6.894757$ & $=1.0 \mathrm{kPa}$ \\
\hline
\end{tabular}

\section{References}

Abdi, M., Moradi, S., Habibnia, B., Kord, S., 2014. Improving oil recovery during water injection and WAG processes in asphaltenic oil reservoirs by using nonionic surfactants. Int. J. Sci. Emerg. Technol. 7 (4), 302-308.

Adebayo, A.R., Barri, A.A., Shahzad Kamal, M., 2017. Effect of flow direction on relative permeability curves in water/gas reservoir system: implications in geological CO2 sequestration. Geofluids https://doi.org/10.1155/2017/1958463, ID-1958463.

Afzali, S., Rezaei, N., Zendehboudi, S., 2018. A comprehensive review on enhanced oil recovery by water alternating gas (WAG) injection. Fuel 227, 218-246. https://doi.org/ 10.1016/j.fuel.2018.04.015.

Alizadeh, A.H., Piri, M., 2014a. Three-phase flow in porous media: a review of experimental studies on relative permeability. Rev. Geophys. 52 (3), 468-521. https://doi.org/ 10.1002/2013RG000433.

Alizadeh, A.H., Piri, M., 2014b. The effect of saturation history on three-phase relative permeability: an experimental study. Water Resour. Res. 50 (2), 1636-1664. https:// doi.org/10.1002/2013WR014914.

Baker, L.E., 1988. Three phase relative permeability correlation, SPE-17369-MS. In: Presented at the SPE/DOE Symposium on Enhanced Oil Recovery, Tulsa, Okla, 17-20 April. https://doi.org/10.2118/17369-MS

Berg, S., Ott, H., 2012. Stability of CO2-brine immiscible displacement. International Journal of Greenhouse Gas Control 11, 188-203. https://doi.org/10.1016/j.ijggc.2012.07. 001.

Bianchi Janetti, E., Riva, M., Guadagnini, A., 2015. Analytical expressions for three-phase generalized relative permeabilities in water- and oil-wet capillary tubes. Comput. Geosci. 20 (3), 555-565. https://doi.org/10.1007/s10596-015-9508-5.

Blunt, M.J., 2017. Multiphase Flow in Permeable Media: A Pore-Scale Perspective. Cambridge University Press.

Blunt, M.J., 2000. An empirical model for three-phase relative permeability. SPE J. 5 (3), 435-445. https://doi.org/10.2118/67950-PA, SPE-56474.

Carlson, F.M., 1981. Simulation of relative permeability hysteresis to the nonwetting phase. In: SPE-10157-MS, Presented at the SPE Annual Technical Conference and Exhibition, San Antonio, Texas, USA, October 5-7. https://doi.org/10.2118/10157-MS.

Caudle, B.H., Slobod, R.L., Brownscombe, E.R., 1951. Further developments in the laboratory determination of relative permeability. J. Petrol. Technol. 3 (5), 145-150. https: //doi.org/10.2118/951145-G, SPE-951145-G.

Chen, B., Reynolds, A.C., 2015. Ensemble-based optimization of the WAG injection process. In: SPE-173217, Presented at the SPE Reservoir Simulation Symposium, Texas, USA, February 23-25. https://doi.org/10.2118/173217-PA

Chen, Z., Huan, G., Ma, Y., 2006. Computational methods for multiphase flows in porous media. In: SIAM Comp. Sci. Eng. Philadelphia. https://doi.org/10.1137/1. 9780898718942.

Christensen, J.R., Stenby, E.H., Skauge, A., 2001. Review of WAG field experience. SPE Reservoir Eval. Eng. 4 (2), 19-23. https://doi.org/10.2118/71203-PA, SPE-71203-PA.

Chukwudeme, E.A., Fjelde, I., Abeysinghe, K.P., Lohne, A., 2014. Effect of interfacial tension on water/oil relative permeability on the basis of history matching to coreflood data. SPE Reservoir Eval. Eng. 17 (1), 37-48. https://doi.org/10.2118/143028-PA, SPE-143028-PA.

Di Carlo, D.A., Sahni, A., Blunt, M.J., 2000. The effect of wettability on three-phase relative permeability. Transport Porous Media 39 (3), 347-366. https://doi.org/10.1023/ A:1006653323374.

Dong, M., Foraie, J., Huang, S., Chatzis, I., 2005. Analysis of immiscible water-alternating-gas (WAG) injection using micromodel tests. J. Can. Petrol. Technol. 44 (2), 17-25. https://doi.org/10.2118/05-02-01.

Egermann, P., Vizika, O., Dallet, L., Requin, C., Sonier, F., 2000. Hysteresis in three-phase flow: experiments, modeling and reservoir simulations. In: SPE-65127-MS, Presented at the SPE European Petroleum Conference, Paris, France, 24-25 October. https://doi. org/10.2118/65127-MS.

Ertekin, T., Abou-Kassen, J.H., King, G.R., 2001. Basic Applied Reservoir Simulation. SPE Textbook Series. Society of Petroleum Engineers, Richardson, Texas.

Fayers, F., Blunt, M., Christie, M., 1991. Accurate calibration of empirical viscous fingering models. Oil Gas Sci. Technol. 46 (3), 311-324. https://doi.org/10.2516/ogst: 1991015.

Fayers, F.J., Matthews, J.D., 1984. Evaluation of normalized stone's methods for estimating three-phase relative permeabilities. SPE J. 24 (02), 224-232. https://doi.org/10. 2118/11277-PA, SPE-11277-PA.

Fenwick, D.H., Blunt, M.J., 1998. Three-dimensional modeling of three-phase imbibition and drainage. J. Adv. Water Resour. 21 (2), 121-143. https://doi.org/10.1016/ S0309-1708(96)00037-1

Floris, F.J.T., Bush, M.D., Cuypers, M., Roggero, F., Syversveen, A.R., 2001. Methods for quantifying the uncertainty of production forecasts: a comparative study. Petrol. Geosci. 7, S87-S96. https://doi.org/10.1144/petgeo.7.S.S87.

Henderson, G., Danesh, A., Tehrani, D., Al-Shaidi, S., Peden, J., 1998. Measurement and correlation of gas condensate relative permeability by the steady-state method. SPE Reservoir Eval. Eng. 1 (2), 134-140. https://doi.org/10.2118/31065-PA, SPE-31065-PA.

Hoseini, J., Masoudi, R., Mirkalaei, M., Mousa, S., Ataei, A., Demiral, B., 2011. Investigating the effects of hysteresis modeling on numerical simulation of immiscible WAG injection. In: IPTC-15055-MS, Presented at the International Petroleum Technology Conference, Bangkok, Thailand, February 15-17. https://doi.org/10.2523/ IPTC-15055-MS.
Jerauld, G.R., 1997. General three-phase relative permeability model for Prudhoe Bay. SPE Reservoir Eng. 12 (4), 255-263. https://doi.org/10.2118/36178-PA, SPE-36178-PA.

Juanes, R., Blunt, M.J., 2006. Impact of viscous fingering on the prediction of optimum WAG ratio. In: SPE-99721-MS, Presented at SPE/DOE Symposium on Improved Oil Recovery, Tulsa, Oklahoma, USA, 22-26 April. https://doi.org/10.2118/99721-MS.

Juanes, R., Patzek, T.W., 2004. Analytical solution to the Riemann problem of three-phase flow in porous media. Transport Porous Media 55 (1), 47-70. https://doi.org/10 1023/B:TIPM.0000007316.43871.1e.

Kalaydjian, F.J.M., Moulu, J.C., Vizika, O., Munkerud, P.K., 1997. Three-phase flow in water-wet porous media: gas/oil relative permeabilities for various spreading conditions. J. Petrol. Sci. Eng. 17 (3), 275-290. https://doi.org/10.1016/ S0920-4105(96)00038-1.

Khorsandi, S., Li, L., Johns, R.T., 2018. A new way of compositional simulation without phase labeling. In: SPE-190269-MS, Presented at the SPE Improved Oil Recovery Conference. Tulsa, Oklahoma, USA, 4-18 April. https://doi.org/10.2118/190269-MS.

Khorsandi, S., Li, L., Johns, R.T., 2017. Equation of state for relative permeability, including hysteresis and wettability alteration. SPE J. 22 (6), 1915-1928. https://doi.org/ 10.2118/182655-PA, SPE-182655-PA.

Kianinejad, A., DiCarlo, D.A., 2016. Three-phase oil relative permeability in water-wet media: a comprehensive study. Transport Porous Media 112 (3), 665-687. https://doi. org/10.1007/s11242-016-0669-z.

Kianinejad, A., Chen, X., Di Carlo, D.A., 2015. The effect of saturation path on three-phase relative permeability. Water Resour. Res. 51 (11), 9141-9164. https://doi.org/10 1002/2015WR017185.

Killough, J.E., 1976. Reservoir simulation with history-dependent saturation functions. SPE J. 16 (1), 37-48. https://doi.org/10.2118/5106-PA, SPE-5106-PA.

Kulkarni, M.M., Rao, D.N., 2005. Experimental investigation of miscible and immiscible water-alternating-gas (WAG) process performance. J. Petrol. Sci. Eng. 48 (1), 1-20. https://doi.org/10.1016/j.petrol.2005.05.001.

Land, C.S., 1968. Calculation of imbibition relative permeability for two- and three-phase flow from rock properties. SPE J. 8 (2), 149-156. https://doi.org/10.2118/1942-PA, SPE-1942-PA.

Larsen, J.A., Skauge, A., 1998. Methodology for numerical simulation with cycle-dependent relative permeabilities. SPE J. 3 (2), 163-173. https://doi.org/10.2118/ 38456-PA, SPE-38456-PA.

Lenhard, R.J., Oostrom, M., 1998. A parametric model for predicting relative permeability-saturation-capillary pressure relationships of oil-water systems in porous media with mixed wettability. Transport Porous Media 31 (1), 109-131. https://doi.org/10. 1023/A:1006503406056.

Lenhard, R.J., Parker, J.C., 1987. A model for hysteretic constitutive relations governing multiphase flow: 2. Permeability-saturation relations. J. Water Resour. Res. 23 (12), 2197-2206. https://doi.org/10.1029/WR023i012p02197.

Lie, K.A., 2014. An Introduction to Reservoir Simulation Using MATLAB: User Guide for the Matlab Reservoir Simulation Toolbox (MRST). https://www.sintef.no/ projectweb/mrst/publications

Lomeland, F., Ebeltoft, E., 2013. Versatile three-phase correlations for relative permeability and capillary pressure. In: SCA2013-034, Presented at the International Symposium of the Society of Core Analysts. Napa Valley, California, USA, pp. 16-19 September.

Moghadasi, L., Guadagnini, A., Inzoli, F., Bartosek, M., Renna, D., 2016. Characterization of two- and three-phase relative permeability of water-wet porous media through X-Ray saturation measurements. J. Petrol. Sci. Eng. 145, 453-463. https://doi.org/10. 1016/j.petrol.2016.05.031.

Oak, M.J., 1990. Three-phase relative permeability of water-wet Berea. SPE-20183-MS. In Presented at the 7th SPE/DOE Enhanced Oil Recovery Symposium, Tulsa, Oklahoma, 22-25 April. https://doi.org/10.2118/20183-MS.

Patel, P., Christman, P., Gardner, J., 1987. Investigation of unexpectedly low field-observed fluid mobilities during some CO2 tertiary floods. SPE Reservoir Eng. 2 (04), 507-513. https://doi.org/10.2118/14308-PA, SPE-14308-PA.

Piri, M., Blunt, M.J., 2005. Three-dimensional mixed-wet random pore-scale network modeling of two and three-phase flow in porous media, I. model description. Phys. Rev. E. 71, 206-301. https://doi.org/10.1103/PhysRevE.71.026301.

Ranaee, E., Inzoli, F., Riva, M., Cominelli, A., Guadagnini, A., 2018a. Propagation to reservoir simulation of uncertainty associated with three-phase relative permeability models with hysteresis. In: SPE-190825-MS, Presented at SPE Europec Featured at 80th EAGE Conference and Exhibition, Copenhagen, Denmark, 11-14 June. https://doi. org/10.2118/190825-MS.

Ranaee, E., Inzoli, F., Riva, M., Maddinelli, G., Cominelli, A., Guadagnini, A., 2018b. Numerical assessment of Water Alternating Gas practices in the presence of hysteresis effects on relative permeability. In: Presented at 16th European Conference on the Mathematics of Oil Recovery, ECMOR XVI, Barcelona, Spain, 3-6 September. https:// doi.org/10.3997/2214-4609.201802255.

Ranaee, E., Moghadasi, L., Inzoli, F., Riva, M., Guadagnini, A., 2017. Identifiability of parameters of three-phase oil relative permeability models under simultaneous water and gas (SWAG) injection. J. Petrol. Sci. Eng. 159, 942-951. https://doi.org/10.1016/ j.petrol.2017.09.062

Ranaee, E., Riva, M., Porta, G.M., Guadagnini, A., 2016. Comparative assessment of three-phase oil relative permeability models. Water Resour. Res. 52 (7), 5341-5356. https://doi.org/10.1002/2016WR018872.

Ranaee, E., Porta, G.M., Riva, M., Blunt, M.J., Guadagnini, A., 2015. Prediction of three-phase oil relative permeability through a sigmoid-based model. J. Petrol. Sci. Eng. 126, 190-200. https://doi.org/10.1016/j.petrol.2014.11.034.

Schlumberger Geo-Quest, 2010. ECLIPSE 100 Reference Manual.

Shahverdi, H., Sohrabi, M., 2013. An improved three-phase relative permeability and hysteresis model for the simulation of a water-alternating-gas injection. SPE J. 18 (5), 841-850. https://doi.org/10.2118/152218-PA, SPE-152218-PA

Shahverdi, H., Sohrabi, M., Fatemi, M., Jamiolahmady, M., 2011. Three-phase relative permeability and hysteresis effect during WAG process in mixed wet and low IFT sys- 
tems. J. Petrol. Sci. Eng. 78 (3), 732-739. https://doi.org/10.1016/j.petrol.2011.08.010. Snell, R.W., 1962. Three-phase relative permeability in an unconsolidated sand. J. Inst. Petrol. 48 (459), 80-88.

Sohrabi, M., Danesh, A., Jamiolahmady, M., 2008. Visualization of residual oil recovery by near-miscible gas and SWAG injection using high-pressure micromodels. Transport Porous Media 74 (2), 239-257. https://doi.org/10.1007/s11242-007-9193-5.

Sohrabi, M., Tehrani, D., Danesh, A., Henderson, G., 2004. Visualization of oil recovery by water-alternating-gas injection using high-pressure micromodels. J. Petrol. Sci. Eng. 9 (03), 290-301. https://doi.org/10.2118/71494-MS, SPE-71494-MS.

Speight, J.G., 2009. Enhanced Recovery Methods for Heavy Oil and Tar Sands. Elsevier.

Spiteri, E.J., Juanes, R., 2006. Impact of relative permeability hysteresis on the numerical simulation of WAG injection. J. Petrol. Sci. Eng. 50 (2), 115-139. https://doi.org/10. 1016/j.petrol.2005.09.004.

Stone, H.L., 1973. Estimation of three-phase relative permeability and residual oil data. J. Can. Petrol. Technol. 12 (4), 53-61. https://doi.org/10.2118/73-04-06, PETSOC-73-04-06.

Stone, H.L., 1970. Probability model for estimation of three-phase relative permeability. J. Petrol. Technol. 22 (2), 214-218. https://doi.org/10.2118/2116-PA, SPE-2116-PA.

Suicmez, V.S., Piri, M., Blunt, M.J., 2008. Effects of wettability and pore-level displacement on hydrocarbon trapping. Adv. Water Resour. 31 (3), 503-512. https://doi.org/ 10.1016/j.advwatres.2007.11.003.

Suicmez, V.S., Piri, M., Blunt, M.J., 2007. Pore-scale simulation of water alternate gas injection. Transport Porous Media 66 (3), 259-286. https://doi.org/10.1007/ s11242-006-0017-9.
Van Dijke, M.I.J., Sorbie, K.S., 2003. Pore-scale modeling of three-phase flow in mixed-wet porous media: multiple displacement chains. J. Petrol. Sci. Eng. 39, 201-216. https:// doi.org/10.1016/S0920-4105(03)00063-9.

Van Dijke, M., Sorbie, K., Sohrabi, M., Tehrani, D., Danesh, A., 2002. Three-phase flow in WAG processes in mixed-wet porous media: pore-scale network simulations and comparison with micromodel experiments, SPE-75192-MS. In: Presented at SPE/DOE Improved Oil Recovery Symposium, Tulsa, Oklahoma, USA, 13-17 April. https://doi. org/10.2118/75192-MS.

Van Dijke, M.I.J., Sorbie, K.S., Sohrabi, M., Danesh, A., 2006. Simulation of WAG floods in an oil-wet micromodel using a 2-D pore-scale network model. J. Petrol. Sci. Eng. 52, 71-86. https://doi.org/10.1016/j.petrol.2006.03.014.

Virnovsky, G.A., Helset, H.M., Skjæveland, S.M., 1994. Stability of displacement fronts in WAG operations. In: SPE-28622-PA, Presented at the SPE Annual Technical Conference and Exhibition, New Orleans, LA, USA, 25-28 September. https://doi.org/10. 2118/28622-PA.

Vizika, O., Lombard, J.M., 1996. Wettability and spreading: two key parameters in oil recovery with three-phase gravity drainage. SPE Reservoir Eng. 11 (1), 54-60. https:// doi.org/10.2118/28613-PA, SPE-28613-PA.

Zhang, Y., Sayegh, S., Huang, S., 2006. Enhanced heavy oil recovery by immiscible WAG injection, PETSOC-2006-014. In: Presented at Canadian International Petroleum Conference, Calgary, Alberta, 13-15 June. https://doi.org/10.2118/2006-014. 